\title{
Spin determination of single-produced resonances at hadron colliders
}

\author{
Yanyan Gao, ${ }^{1,2}$ Andrei V. Gritsan, ${ }^{1}$ Zijin Guo, ${ }^{1}$ Kirill Melnikov, ${ }^{1}$ Markus Schulze, ${ }^{1}$ and Nhan V. Tran ${ }^{1}$ \\ ${ }^{1}$ Department of Physics and Astronomy, Johns Hopkins University, Baltimore, MD, USA \\ ${ }^{2}$ Fermi National Accelerator Laboratory (FNAL), Batavia, IL, USA \\ (Dated: submitted on January 19, 2010; revised on March 12, 2010)
}

\begin{abstract}
We study the production of a single resonance at the LHC and its decay into a pair of $Z$ bosons. We demonstrate how full reconstruction of the final states allows us to determine the spin and parity of the resonance and restricts its coupling to vector gauge bosons. Full angular analysis is illustrated with the simulation of the production and decay chain including all spin correlations and the most general couplings of spin-zero, -one, and -two resonances to Standard Model matter and gauge fields. We note implications for analysis of a resonance decaying to other final states.
\end{abstract}

PACS numbers: 12.60.-i, 13.88.+e, 14.80.Bn

\section{INTRODUCTION}

Physics beyond the Standard Model (SM), to be probed at the LHC [1 3 ], will manifest itself through observations of new particles. Such observations are instrumental for establishing the existence of New Physics, though more effort is required to understand these observations in detail. It will be crucial to determine the quantum numbers of the new particles, their masses, and their couplings to SM fields as accurately as possible.

Measuring masses, coupling constants, and quantum numbers at a hadron collider is difficult, though many techniques for doing so were put forward recently. Some of those techniques evolved remarkably over time. For example, top quark mass determinations at the Tevatron [4] started out from measurements of the $t \bar{t}$ production cross-section and establishing the value of $m_{t}$ which fits the cross-section best. A more recent technique - "the matrix element method" - performs a likelihood fit on an event-by-event basis. Since more information about the event is used, more efficient separation of signal and background is accomplished and a higher accuracy of the top quark mass measurement is achieved.

The idea that matrix elements, or multivariate per-event likelihoods, can guide us in maximizing the amount of information that can be extracted from a given event is appealing; but, to the best of our knowledge, it has not been widely used in hadron collider physics beyond top mass determinations. On the other hand, these techniques are not new to experimental analyses since they were used in many $B$-physics measurements [5].

The goal of this paper is to apply the multivariate likelihood method to the determination of a spin of a resonance, produced in hadron collisions. Many extensions of the SM postulate the existence of (elementary) particles of different spins that can be single-produced at the LHC. Once produced, these resonances decay into SM particles whose angular distributions contain information about couplings, spins, and other quantum numbers of their parents.

Spin determination is often discussed in the context of a particular angular distribution; the challenge is to find a distribution that exhibits maximal sensitivity to the spin of a resonance. Single-observable distributions may be viable spin-analyzers but, as we illustrate with some examples in this paper, loss of statistical power and certain information is inevitable. Construction of the likelihood of the hypothesis that a given event, with its complete kinematic dependence, comes from the production and decay of a resonance with a particular spin is the most efficient way to analyze the events. Testing this approach in a realistic hadron collider setting is what we would like to do in this paper.

It is best to pursue this program in a situation where the final state is reconstructed fully and accurately. For this reason we exclude the final states with missing energy and jets and examine the pure leptonic final states. It follows that we can either consider direct decays of resonances to a lepton pair or we can look at the decays of such resonances into neutral gauge bosons that subsequently decay into leptons. There are three reasons for us to choose the second option. First, more information can be extracted from a fully-reconstructed four-body final state [6- 12] than from a two-body final state; second, direct decays to $l^{+} l^{-}$are studied well in the literature $[13$ 18]; and, third, it is reasonable to assume that the decay of a single-produced resonance to $Z$ bosons is sizable, if not altogether dominant. Recall that this happens with the SM Higgs boson if its mass exceeds $2 m_{z}$ [19]. It may also occur in wellmotivated scenarios of Beyond the Standard Model (BSM) physics. For example, in the extra-dimensional model [20]

${ }^{1}$ In the appendix we present angular distributions for $X \rightarrow l^{+} l^{-}$that generalize results in the literature. 


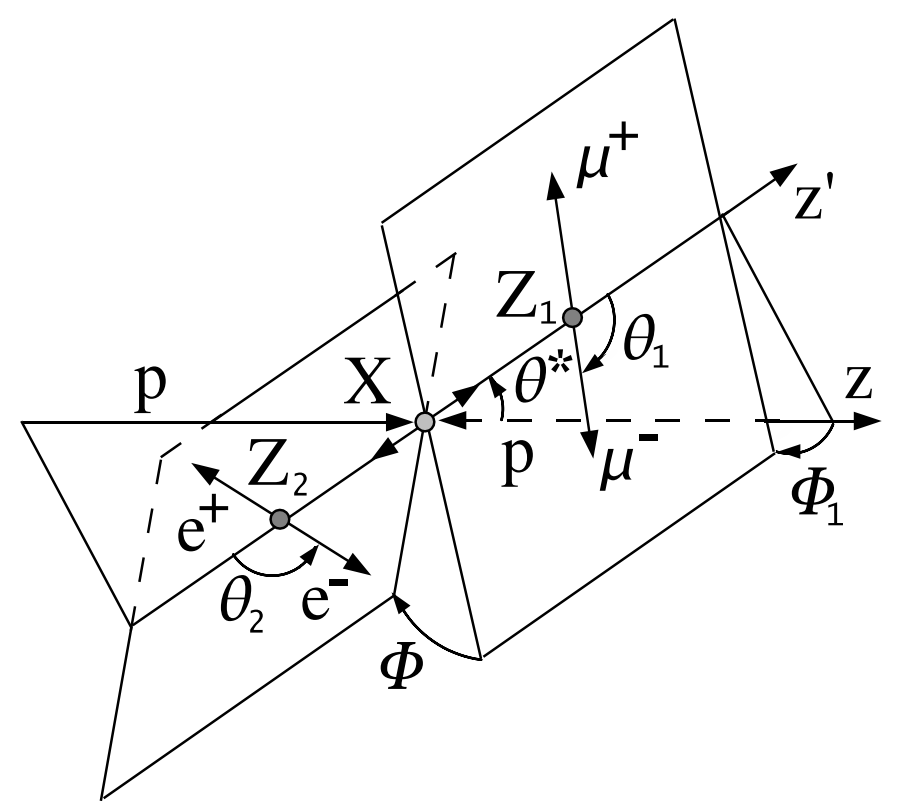

FIG. 1: Illustration of an exotic $X$ particle production and decay in $p p$ collision $g g$ or $q \bar{q} \rightarrow X \rightarrow Z Z \rightarrow 4 l^{ \pm}$. Six angles fully characterize orientation of the decay chain: $\theta^{*}$ and $\Phi^{*}$ of the first $Z$ boson in the $X$ rest frame, two azimuthal angles $\Phi$ and $\Phi_{1}$ between the three planes defined in the $X$ rest frame, and two $Z$-boson helicity angles $\theta_{1}$ and $\theta_{2}$ defined in the corresponding $Z$ rest frames. The offset of angle $\Phi^{*}$ is arbitrarily defined and therefore this angle is not shown.

discussed in Refs. 21 23] KK graviton decays into pairs of gauge bosons are enhanced relative to direct decays into leptons. Similar situations may occur in "hidden-valley"-type models 24]. An example of a "heavy photon" is given in Ref. [25].

Motivated by this, we consider the production of a resonance $X$ at the LHC in gluon-gluon and quark-antiquark partonic collisions, with the subsequent decay of $X$ into two $Z$ bosons which, in turn, decay leptonically. In Fig. 1, we show the decay chain $X \rightarrow Z Z \rightarrow e^{+} e^{-} \mu^{+} \mu^{-}$. However, our analysis is equally applicable to any combination of decays $Z \rightarrow e^{+} e^{-}$or $\mu^{+} \mu^{-}$. It may also be applicable to $Z$ decays into $\tau$ leptons since $\tau$ 's from $Z$ decays will often be highly boosted and their decay products collimated. We study how the spin and parity of $X$, as well as information on its production and decay mechanisms, can be extracted from angular distributions of four leptons in the final state.

There are a few things that need to be noted. First, we obviously assume that the resonance production and its decays into four leptons are observed. Note that, because of a relatively small branching fraction for leptonic $Z$ decays, this assumption implies a fairly large production cross-section for $p p \rightarrow X$ and a fairly large branching fraction for the decay $X \rightarrow Z Z$. As we already mentioned, there are well-motivated scenarios of BSM physics where those requirements are satisfied.

Second, having no bias towards any particular model of BSM physics, we consider the most general couplings of the particle $X$ to relevant SM fields. This approach has to be contrasted with typical studies of e.g. spin-two particles at hadron colliders where such an exotic particle is often identified with a massive graviton that couples to SM fields through the energy-momentum tensor. We will refer to this case as the "minimal coupling" of the spin-two particle to SM fields.

The minimal coupling scenarios are well-motivated within particular models of New Physics, but they are not sufficiently general. For example, such a minimal coupling may restrict partial waves that contribute to the production and decay of a spin-two particle. Removing such restriction opens an interesting possibility to understand the couplings of a particle $X$ to SM fields by means of partial wave analyses, and we would like to set a stage for doing that in this paper. To pursue this idea in detail, the most general parameterization of the $X$ coupling to SM fields is required. Such parameterizations are known for spin-zero, spin-one, and spin-two particles interacting with the SM gauge bosons 7,8 and we use these parameterizations in this paper. We also note that the model recently discussed in Refs. 21 23] requires couplings beyond the minimal case in order to produce longitudinal polarization dominance.

Third, we note that while we concentrate on the decay $X \rightarrow Z Z \rightarrow l_{1}^{+} l_{1}^{-} l_{2}^{+} l_{2}^{-}$, the technique discussed in this paper is more general and can, in principle, be applied to final states with jets and/or missing energy by studying such processes as $X \rightarrow Z Z \rightarrow l^{+} l^{-} j j, X \rightarrow W^{+} W^{-} \rightarrow l^{+} \nu j j$, etc. In contrast with pure leptonic final states, higher statistics, larger backgrounds, and a worse angular resolution must be expected once final states with jets and 
missing energy are included. We plan to perform detailed studies of these, more complicated final states, in the future. However, we note that many results in this paper are applicable to these final states as well.

The remainder of the paper is organized as follows. In Section II, we describe the parameterization of production and decay amplitudes that is employed in our analyses. In Section IIII, we calculate helicity amplitudes for the decay of a resonance into a pair of gauge bosons or into a fermion-antifermion pair; helicity amplitudes for resonance production are obtained by crossing. In Section [V] angular distributions for $p p \rightarrow X \rightarrow Z Z \rightarrow f_{1} \bar{f}_{1} f_{2} \bar{f}_{2}$ for resonances with spins zero, one, and two are presented. This is followed by detailed Monte Carlo simulation which includes all spin correlations and main experimental effects and which is shown in Section $\nabla$. Analysis using the multivariate maximum likelihood technique is applied to several key scenarios to illustrate separation power of different helicity amplitudes for all spin hypotheses and in both production and decay, as discussed in Section VI] For completeness, angular distributions, including distributions for other decay channels, are given in the appendix.

\section{INTERACTIONS OF AN EXOTIC PARTICLE WITH STANDARD MODEL FIELDS}

In this section, the interaction of a color- and charge-neutral exotic particle $X$ with two spin-one bosons $V$ (such as gluons, photons, $Z$, or $W$ bosons) or a fermion-antifermion pair (such as leptons or quarks) is summarized. The spin of $X$ can be zero, one, or two. We construct the most general amplitudes consistent with Lorentz invariance and Bose-symmetry, as well as gauge-invariance with respect to unbroken subgroups of $S U(3) \times S U(2)_{L} \times U(1)_{R}$ of the SM.

The four-momentum of the particle $X$ is denoted by $q$ and the four-momenta of the gauge bosons or fermions by $q_{1,2}$. The polarization vectors of gauge bosons are denoted by $\epsilon_{1,2}$; we assume them to be transverse $q_{i} \epsilon_{i}=0$. Fermion wave functions are conventional Dirac spinors. We employ the field strength tensor of a gauge boson with momentum $q_{i}$ and polarization vector $\epsilon_{i}$ as $f^{(i), \mu \nu}=\epsilon_{i}^{\mu} q_{i}^{\nu}-\epsilon_{i}^{\nu} q_{i}^{\mu}$, and the conjugate field strength tensor as $\tilde{f}_{\mu \nu}^{(i)}=$ $1 / 2 \epsilon_{\mu \nu \alpha \beta} f^{(i), \alpha \beta}=\epsilon_{\mu \nu \alpha \beta} \epsilon_{i}^{\alpha} q_{i}^{\beta}$. We use $\tilde{q}=q_{1}-q_{2}$ to denote the particular combination of the momenta of the two final state particles.

\section{A. Spin-zero $X$ and two gauge bosons}

The invariant amplitude that describes the interaction between a spin-zero particle $X$ of arbitrary parity and two spin-one gauge bosons reads

$$
A(X \rightarrow V V)=v^{-1}\left(g_{1}^{(0)} m_{V}^{2} \epsilon_{1}^{*} \epsilon_{2}^{*}+g_{2}^{(0)} f_{\mu \nu}^{*(1)} f^{*(2), \mu \nu}+g_{3}^{(0)} f^{*(1), \mu \nu} f_{\mu \alpha}^{*(2)} \frac{q_{\nu} q^{\alpha}}{\Lambda^{2}}+g_{4}^{(0)} f_{\mu \nu}^{*(1)} \tilde{f}^{*(2), \mu \nu}\right) .
$$

In Eq. (1), $f^{*}$ denotes the complex conjugate field strength tensor, $v$ is the SM vacuum expectation value of the Higgs field, and $\Lambda$ is the mass scale associated with BSM physics. The "couplings" $g_{1, \ldots, 4}^{(0)}$ are invariant form-factors; the upper index reflects the $X$ spin. Since we consider on-shell decays of the particle $X$ to two on-shell gauge-bosons, $g_{j}^{(i)}$ can be thought of as effective dimensionless coupling constants which can, in general, be complex.

We note that, as written, Eq. (1) does not use the minimal set of independent variables since it uses both, the field strength tensors and polarization vectors for gauge bosons in the final state. However, we write Eq. (1) in that particular way because it can be applied to $X$ decays into both massive and massless gauge bosons and because it has the simplest possible connection to SM couplings at tree level. Indeed, if we identify $X$ with the Higgs boson of the SM, the proper tree-level amplitude for $H \rightarrow Z Z$ is obtained by setting $g_{j>1}^{(0)}=0$ and $g_{1}^{(0)}=2 i$. To describe the coupling of the spin-zero particle to massless gauge bosons (gluons or photons), we simply $\operatorname{set}^{2} m_{V}=0$ in Eq. (1). Clearly, the coefficients $g_{j}^{(0), g g}$ for interaction with gluons, for example, do not need to be equal to the coefficients for interaction with the $\mathrm{Z}$ bosons $g_{j}^{(0), z z}$ or the photons $g_{j}^{(0), \gamma \gamma}$. In fact, Eq. (1) is sufficiently general to accommodate all radiative corrections to Higgs interactions with gauge bosons, massive or massless, in the SM, including $C P$-violating form factors that appear at the three loop level [6].

In spite of the fact that there are four form-factors required to describe the interaction of the spin-zero boson with two massive or massless spin-one bosons, there are only three independent structures in the scattering amplitude. To

\footnotetext{
${ }^{2}$ For X coupling to two gluons, a trivial color factor needs to be introduced in Eq. (1).
} 
see this, we rewrite Eq. (1) through polarization vectors

$$
A(X \rightarrow V V)=v^{-1} \epsilon_{1}^{* \mu} \epsilon_{2}^{* \nu}\left(a_{1} g_{\mu \nu} m_{X}^{2}+a_{2} q_{\mu} q_{\nu}+a_{3} \epsilon_{\mu \nu \alpha \beta} q_{1}^{\alpha} q_{2}^{\beta}\right)
$$

and find the coefficients $a_{1,2,3}$ to be

$$
a_{1}=g_{1}^{(0)} \frac{m_{V}^{2}}{m_{X}^{2}}+g_{2}^{(0)} \frac{2 s}{m_{X}^{2}}+g_{3}^{(0)} \kappa \frac{s}{m_{X}^{2}}, \quad a_{2}=-2 g_{2}^{(0)}-g_{3}^{(0)} \kappa, \quad a_{3}=-2 g_{4}^{(0)} .
$$

We have defined the parameters $s=q_{1} q_{2}=\left(m_{X}^{2}-2 m_{V}^{2}\right) / 2$ and $\kappa=s / \Lambda^{2}$. The amplitude for $X$ decay into two massless gauge bosons is obtained from Eqs. (2) and (3) by setting $m_{V}$ to zero.

\section{B. Spin-one $X$ and two gauge bosons}

We consider the case when the exotic particle $X$ has spin one and arbitrary parity. As a consequence of the LandauYang theorem, the spin-one particle $X$ cannot interact with two massless identical gauge bosons. For this reason, a spin-one color-singlet particle cannot be produced in gluon fusion, or decay to two photons. The phenomenology of spin-one decays into two $Z$ bosons was recently discussed in Ref. [9]. Following that reference, we consider the amplitude for the decay to two identical massive gauge bosons $X \rightarrow Z Z$. This amplitude depends on two independent form factors

$$
A(X \rightarrow Z Z)=g_{1}^{(1)}\left[\left(\epsilon_{1}^{*} q\right)\left(\epsilon_{2}^{*} \epsilon_{X}\right)+\left(\epsilon_{2}^{*} q\right)\left(\epsilon_{1}^{*} \epsilon_{X}\right)\right]+g_{2}^{(1)} \epsilon_{\alpha \mu \nu \beta} \epsilon_{X}^{\alpha} \epsilon_{1}^{*, \mu} \epsilon_{2}^{*, \nu} \tilde{q}^{\beta}
$$

Similar to the spin-zero case, $g_{1}^{(1)}$ and $g_{2}^{(1)}$ are dimensionless effective coupling constants. We note that these coupling constants are, in general, complex with absorptive parts that may arise from quantum loop effects. This possibility was not considered in Ref. [9] where the case of zero complex phase difference between the two coupling constants was studied. In the case when $X$ has positive parity $\left(J^{P}=1^{+}\right)$, the first term violates and the second term conserves parity. Alternatively, the two terms correspond to parity-conserving and parity-violating interactions of the $1^{-}$ particle, respectively.

\section{Spin-two $X$ and two gauge bosons}

We turn to the spin-two case and construct the most general amplitude for the decay of a spin-two particle $X$ into two identical vector gauge bosons. The $X$ wave function is given by a symmetric traceless tensor $t_{\mu \nu}$, transverse to its momentum $t_{\mu \nu} q^{\nu}=0$. Since we would like to apply the formula for the amplitude to describe interactions of $X$ with massive and massless gauge bosons, we consider the possible dependence of the amplitude on both the field strength tensor and the polarization vectors

$$
\begin{aligned}
& A(X \rightarrow V V)=\Lambda^{-1}\left[2 g_{1}^{(2)} t_{\mu \nu} f^{* 1, \mu \alpha} f^{* 2, \nu \alpha}+2 g_{2}^{(2)} t_{\mu \nu} \frac{q_{\alpha} q_{\beta}}{\Lambda^{2}} f^{* 1, \mu \alpha} f^{* 2, \nu, \beta}\right. \\
& +g_{3}^{(2)} \frac{\tilde{q}^{\beta} \tilde{q}^{\alpha}}{\Lambda^{2}} t_{\beta \nu}\left(f^{* 1, \mu \nu} f_{\mu \alpha}^{* 2}+f^{* 2, \mu \nu} f_{\mu \alpha}^{* 1}\right)+g_{4}^{(2)} \frac{\tilde{q}^{\nu} \tilde{q}^{\mu}}{\Lambda^{2}} t_{\mu \nu} f^{* 1, \alpha \beta} f_{\alpha \beta}^{*(2)} \\
& +m_{V}^{2}\left(2 g_{5}^{(2)} t_{\mu \nu} \epsilon_{1}^{* \mu} \epsilon_{2}^{* \nu}+2 g_{6}^{(2)} \frac{\tilde{q}^{\mu} q_{\alpha}}{\Lambda^{2}} t_{\mu \nu}\left(\epsilon_{1}^{* \nu} \epsilon_{2}^{* \alpha}-\epsilon_{1}^{* \alpha} \epsilon_{2}^{* \nu}\right)+g_{7}^{(2)} \frac{\tilde{q}^{\mu} \tilde{q}^{\nu}}{\Lambda^{2}} t_{\mu \nu} \epsilon_{1}^{*} \epsilon_{2}^{*}\right) \\
& \left.+g_{8}^{(2)} \frac{\tilde{q}_{\mu} \tilde{q}_{\nu}}{\Lambda^{2}} t_{\mu \nu} f^{* 1, \alpha \beta} \tilde{f}_{\alpha \beta}^{*(2)}+g_{9}^{(2)} t_{\mu \alpha} \tilde{q}^{\alpha} \epsilon_{\mu \nu \rho \sigma} \epsilon_{1}^{* \nu} \epsilon_{2}^{* \rho} q^{\sigma}+\frac{g_{10}^{(2)} t_{\mu \alpha} \tilde{q}^{\alpha}}{\Lambda^{2}} \epsilon_{\mu \nu \rho \sigma} q^{\rho} \tilde{q}^{\sigma}\left(\epsilon_{1}^{* \nu}\left(q \epsilon_{2}^{*}\right)+\epsilon_{2}^{* \nu}\left(q \epsilon_{1}^{*}\right)\right)\right]
\end{aligned}
$$

As in the spin-zero and spin-one cases, $g_{1, \ldots, 10}^{(2)}$ are dimensionless effective coupling constants which are, in general, complex numbers. They are different for different gauge bosons $V$. The first seven constants $g_{1, \ldots, 7}^{(2)}$ correspond to the $J^{P}=2^{+}$particle parity-conserving interaction, while the last three terms with $g_{8,9,10}^{(2)}$ correspond to its parity-violating interaction. Alternatively, they correspond to parity-violating and parity-conserving interactions of the $2^{-}$particle, respectively. 
We can now write the amplitude through polarization vectors

$$
\begin{aligned}
& A(X \rightarrow Z Z)=\Lambda^{-1} e_{1}^{* \mu} e_{2}^{* \nu}\left[c_{1}\left(q_{1} q_{2}\right) t_{\mu \nu}+c_{2} g_{\mu \nu} t_{\alpha \beta} \tilde{q}^{\alpha} \tilde{q}^{\beta}+c_{3} \frac{q_{2 \mu} q_{1 \nu}}{m_{X}^{2}} t_{\alpha \beta} \tilde{q}^{\alpha} \tilde{q}^{\beta}+2 c_{4}\left(q_{1 \nu} q_{2}^{\alpha} t_{\mu \alpha}\right.\right. \\
& \left.\left.+q_{2 \mu} q_{1}^{\alpha} t_{\nu \alpha}\right)+c_{5} t_{\alpha \beta} \frac{\tilde{q}^{\alpha} \tilde{q}^{\beta}}{m_{X}^{2}} \epsilon_{\mu \nu \rho \sigma} q_{1}^{\rho} q_{2}^{\sigma}+c_{6} t^{\alpha \beta} \tilde{q}_{\beta} \epsilon_{\mu \nu \alpha \rho} q^{\rho}+\frac{c_{7} t^{\alpha \beta} \tilde{q}_{\beta}}{m_{X}^{2}}\left(\epsilon_{\alpha \mu \rho \sigma} q^{\rho} \tilde{q}^{\sigma} q_{\nu}+\epsilon_{\alpha \nu \rho \sigma} q^{\rho} \tilde{q}^{\sigma} q_{\mu}\right)\right] .
\end{aligned}
$$

The coefficients $c_{1-7}$ can be expressed through $g_{1, \ldots, 10}^{(2)}$

$$
\begin{aligned}
& c_{1}=2 g_{1}^{(2)}+2 g_{2}^{(2)} \kappa\left(1+\frac{m_{V}^{2}}{s}\right)^{2}+2 g_{5}^{(2)} \frac{m_{V}^{2}}{s}, \\
& c_{2}=-\frac{g_{1}^{(2)}}{2}+g_{3}^{(2)} \kappa\left(1-\frac{m_{V}^{2}}{s}\right)+2 g_{4}^{(2)} \kappa+g_{7}^{(2)} \kappa \frac{m_{V}^{2}}{s}, \\
& c_{3}=-\left(\frac{g_{2}^{(2)}}{2}+g_{3}^{(2)}+2 g_{4}^{(2)}\right) \kappa \frac{m_{X}^{2}}{s}, \\
& c_{4}=-g_{1}^{(2)}-g_{2}^{(2)} \kappa-\left(g_{2}^{(2)}+g_{3}^{(2)}+g_{6}^{(2)}\right) \kappa \frac{m_{V}^{2}}{s}, \\
& c_{5}=2 g_{8}^{(2)} \kappa \frac{m_{X}^{2}}{s}, \quad c_{6}=g_{9}^{(2)}, \quad c_{7}=g_{10}^{(2)} \kappa \frac{m_{X}^{2}}{s} .
\end{aligned}
$$

To describe production of the particle $X$ in hadron collisions, we need to know the $X$ 's coupling to gluons. The corresponding amplitude can be obtained from the case $A(X \rightarrow V V)$ that we just considered by crossing transformation and setting $m_{V}=0, g_{9}^{(2)}=0$. Also, because $e_{1} q_{2}=e_{2} q_{1}=0$ in the massless case, we find that terms proportional to $c_{3}$ and $c_{4}$ do not contribute when an analog of Eq. (6) is written for massless gauge bosons.

\section{D. $X$ and two fermions}

For completeness, we also give here the general couplings of the particle $X$ to two fermions. We denote fermion masses as $m_{q}$. We assume that the chiral symmetry is exact in the limit when fermion masses vanish. We obtain the following amplitudes

$$
\begin{aligned}
& A\left(X_{J=0} \rightarrow q \bar{q}\right)=\frac{m_{q}}{v} \bar{u}_{q_{1}}\left(\rho_{1}^{(0)}+\rho_{2}^{(0)} \gamma_{5}\right) v_{q_{2}}, \\
& A\left(X_{J=1} \rightarrow q \bar{q}\right)=\epsilon^{\mu} \bar{u}_{q_{1}}\left(\gamma_{\mu}\left(\rho_{1}^{(1)}+\rho_{2}^{(1)} \gamma_{5}\right)+\frac{m_{q} \tilde{q}_{\mu}}{\Lambda^{2}}\left(\rho_{3}^{(1)}+\rho_{4}^{(1)} \gamma_{5}\right)\right) v_{q_{2}}, \\
& A\left(X_{J=2} \rightarrow q \bar{q}\right)=\frac{1}{\Lambda} t^{\mu \nu} \bar{u}_{q_{1}}\left(\gamma_{\mu} \tilde{q}_{\nu}\left(\rho_{1}^{(2)}+\rho_{2}^{(2)} \gamma_{5}\right)+\frac{m_{q} \tilde{q}_{\mu} \tilde{q}_{\nu}}{\Lambda^{2}}\left(\rho_{3}^{(2)}+\rho_{4}^{(2)} \gamma_{5}\right)\right) v_{q_{2}},
\end{aligned}
$$

where $m_{q}$ is the fermion mass and $\bar{u}$ and $v$ are the Dirac spinors. It follows that, in the case when fermions are massless, the minimal couplings are also the most general ones and no new structures appear.

\section{HELICITY AMPLITUDES}

We are now in position to compute helicity amplitudes for the production and decay processes. Helicity amplitudes are important because, as we will see in the following discussion, those amplitudes parameterize angular distributions and, hence, can be directly extracted from data. By knowing how these amplitudes are expressed through effective couplings introduced in the previous section, we can constrain those couplings through measurements of angular distributions.

To compute the helicity amplitudes $A_{\lambda_{1} \lambda_{2}}$ for the decay $X \rightarrow V V$, we calculate amplitudes presented in the previous section for polarization vectors that correspond to $\lambda_{1}, \lambda_{2}$. We begin with the description of the polarization vectors that we use in the analysis. Consider the decay $X \rightarrow V V$ in the rest frame of $X$. The momenta of the two $V$ 's are parameterized as $q_{1,2}=\left(m_{X} / 2,0,0, \pm \beta m_{X} / 2\right)$, where $\beta=\left(1-4 m_{V}^{2} / m_{X}^{2}\right)^{1 / 2}$ is the velocity of gauge bosons in the $X$ rest frame. The polarization vectors for the two $Z$-bosons read

$$
e_{1,2}^{\mu}(0)=m_{V}^{-1}\left( \pm \beta m_{X} / 2,0,0, m_{X} / 2\right), \quad e_{1}^{\mu}( \pm)=e_{2}^{\mu}(\mp)=\frac{1}{\sqrt{2}}(0, \mp 1,-i, 0) .
$$


The polarization vectors of the particle $X$ are defined as follows. For the spin-one boson, $J_{X}=1$, we use

$$
e_{X}(0)=(0,0,0,1), \quad e_{X}( \pm)=\frac{1}{\sqrt{2}}(0, \mp 1,-i, 0) .
$$

For the spin-two boson, $J_{X}=2$, the polarization vectors read

$$
\begin{aligned}
& t^{\mu \nu}( \pm 2)=e_{X}^{\mu}( \pm) e_{X}^{\nu}( \pm), \quad t^{\mu \nu}( \pm 1)=\frac{1}{\sqrt{2}}\left[e_{X}^{\mu}( \pm) e_{X}^{\nu}(0)+e_{X}^{\mu}(0) e_{X}^{\nu}( \pm)\right], \\
& t^{\mu \nu}(0)=\frac{1}{\sqrt{6}}\left[e_{X}^{\mu}(+) e_{X}^{\nu}(-)+e_{X}^{\mu}(-) e_{X}^{\nu}(+)\right]+\sqrt{\frac{2}{3}} e_{X}^{\mu}(0) e_{X}^{\nu}(0) .
\end{aligned}
$$

It is straightforward to establish general properties of the helicity amplitudes $A_{\lambda_{1} \lambda_{2}}$. In general there are nine complex amplitudes $A_{\lambda_{1} \lambda_{2}}$, since conservation of the angular momentum component along the decay axis fixes the spin projection of the $X$ particle to $\lambda_{X}=\lambda_{1}-\lambda_{2}$. Because of this identity, we do not reference $\lambda_{X}$ in the notation for helicity amplitudes. Moreover, in the case of two identical vector bosons, such as $Z Z, g g$, or $\gamma \gamma$, the number of independent amplitudes is reduced from nine to six due to the following identity [26, 27]

$$
A_{\lambda_{1} \lambda_{2}}=(-1)^{J} A_{\lambda_{2} \lambda_{1}},
$$

where $J$ is the spin of the $X$ particle. If parity is conserved, further constraints apply [26, 27]

$$
A_{\lambda_{1} \lambda_{2}}=\eta_{P}(-1)^{J} A_{-\lambda_{1}-\lambda_{2}},
$$

where $\eta_{P}$ is the parity of the $X$ particle. We note that Eqs. (14, 15) depend on phase conventions for the polarization vectors in Eqs. (11, 12).

For a spin-zero $X$ particle, only $\lambda_{1}-\lambda_{2}=0$ values are possible. Therefore only $A_{++}, A_{--}$, and $A_{00}$ contribute. For a parity-even scalar with $J^{P}=0^{+}$, such as a SM Higgs, one has $A_{++}=A_{--}$and for parity-odd pseudo-scalar with $J^{P}=0^{-}$one has $A_{++}=-A_{--}$and $A_{00}=0$. For a spin-one $X$ particle, Bose symmetry prohibits $A_{++}, A_{--}$, and $A_{00}$ amplitudes, as also evident from Eq. (14). Therefore we are left with only two independent contributions $A_{+0}=-A_{0+}$ and $A_{-0}=-A_{0-}$. Furthermore, in the vector case $J^{P}=1^{-}$, one has $A_{+0}=A_{-0}=-A_{0+}=-A_{0-}$, and for an axial vector $J^{P}=1^{+}$the amplitudes are related as $A_{+0}=-A_{-0}=-A_{0+}=A_{0-}$ in the case of parityconserving interactions. For a spin-two $X$ particle, there are generally six independent contributions $A_{00}, A_{++}$, $A_{--}, A_{+-}=A_{-+}, A_{+0}=A_{0+}$, and $A_{-0}=A_{0-}$. With parity conservation, there are additional constraints for the case $J^{P}=2^{+}: A_{++}=A_{--}$and $A_{+0}=A_{0+}=A_{-0}=A_{0-}$; and for the case $J^{P}=2^{-}: A_{++}=-A_{--}$and $A_{+0}=A_{0+}=-A_{-0}=-A_{0-}$, while $A_{00}=0$ and $A_{+-}=A_{-+}=0$. We now use explicit expressions for the amplitudes constructed in the previous section to illustrate these assertions and compute the independent helicity amplitudes explicitly.

\section{A. Helicity amplitudes for spin-zero decay}

We use Eq. (2) to compute the helicity amplitudes and obtain for the decay to two massive vector bosons

$$
\begin{aligned}
& A_{00}=-\frac{m_{X}^{4}}{4 v m_{V}^{2}}\left(a_{1}\left(1+\beta^{2}\right)+a_{2} \beta^{2}\right), \\
& A_{++}=\frac{m_{X}^{2}}{v}\left(a_{1}+\frac{i a_{3} \beta}{2}\right), \\
& A_{--}=\frac{m_{X}^{2}}{v}\left(a_{1}-\frac{i a_{3} \beta}{2}\right) .
\end{aligned}
$$

As expected, these amplitudes satisfy Eq. (15) for parity-even $\left(a_{1}\right.$ and $\left.a_{2}\right)$ and parity-odd $\left(a_{3}\right)$ contributions separately.

Helicity amplitudes for the decay of the $X$ particle into two massless gauge bosons can be obtained from Eq. (16) by disregarding $A_{00}$, since massless gauge bosons cannot be longitudinally polarized, and by setting $\beta=1$ in $A_{++}$ and $A_{--}$. The relationship between coefficients $a_{1}, a_{3}$ and the fundamental couplings $g_{i}^{(0)}$, in this case, can be read off from Eq. (3), where the mass of the vector boson $m_{V}$ should be set to zero.

It is interesting to point out some features of Eq. (16) for the case when $X$ is the SM Higgs boson, sufficiently heavy to decay into two $Z$ bosons. Then, as follows from Eq. (16) the longitudinal amplitude $A_{00}$ dominates in the limit 
that $m_{H} \gg m_{Z}$. To see this, recall that at tree level in the SM only the term with $a_{1}$ in Eq. (2) contributes. Then, $A_{++}=A_{--}=-A_{00} / \gamma$, where $\gamma$ is the boost between the rest frames of the two $Z$ boson

$$
\gamma=\frac{m_{x}^{2}}{2 m_{Z}^{2}}-1=\frac{1+\beta^{2}}{1-\beta^{2}} .
$$

Note also that additional contributions to the helicity amplitudes are present even in the SM, beyond the tree level. For example, both $a_{2}$ and $a_{3}$ may appear from radiative corrections. The natural scale for $a_{2}$, generated radiatively in the SM, is $\mathcal{O}\left(\alpha_{\mathrm{EW}}\right) \sim 10^{-2}$, while the SM contribution to $a_{3}$ appears only at three loops and therefore is tiny $\mathcal{O}\left(10^{-11}\right)$ [6]. In general, all three coefficients $a_{1}, a_{2}$, and $a_{3}$ are complex numbers with a priori unknown relative complex phases between them. In the context of the SM, the measurement of the radiatively induced contributions $a_{2,3}$ is a non-trivial test of the Higgs couplings to gauge bosons at the quantum level.

\section{B. Helicity amplitudes for spin-one decays}

Using Eq. (4) we find the following helicity amplitudes

$$
\begin{aligned}
& A_{+0}=-A_{0+}=\frac{\beta m_{X}^{2}}{2 m_{z}}\left(g_{1}^{(1)}+i \beta g_{2}^{(1)}\right), \\
& A_{-0}=-A_{0-}=\frac{\beta m_{X}^{2}}{2 m_{Z}}\left(g_{1}^{(1)}-i \beta g_{2}^{(1)}\right) .
\end{aligned}
$$

These amplitudes satisfy Eqs. (14, 15) for parity-even $\left(g_{1}^{(1)}\right)$ and parity-odd $\left(g_{2}^{(1)}\right)$ contributions separately. Note that the Landau-Yang theorem forbids decays of spin-one particles into a pair of massless identical bosons. This feature is apparent from Eq. (18) which shows that one of the vector bosons $V$ in the decay of the spin-one particle $X$ should be longitudinally polarized. Since massless vector bosons cannot be polarized longitudinally, the decay of $X$ into a pair of massless identical vector bosons cannot occur.

\section{Helicity amplitudes for spin-two decays}

Using explicit parameterization for the decay $X \rightarrow V V$ in Eq. (6), we obtain the helicity amplitudes

$$
\begin{aligned}
& A_{+-}=A_{-+}=\frac{m_{X}^{2}}{4 \Lambda} c_{1}\left(1+\beta^{2}\right), \\
& A_{++}=\frac{m_{X}^{2}}{\sqrt{6} \Lambda}\left[\frac{c_{1}}{4}\left(1+\beta^{2}\right)+2 c_{2} \beta^{2}+i \beta\left(c_{5} \beta^{2}-2 c_{6}\right)\right], \\
& A_{--}=\frac{m_{X}^{2}}{\sqrt{6} \Lambda}\left[\frac{c_{1}}{4}\left(1+\beta^{2}\right)+2 c_{2} \beta^{2}-i \beta\left(c_{5} \beta^{2}-2 c_{6}\right)\right], \\
& A_{+0}=A_{0+}=\frac{m_{X}^{3}}{m_{V} \sqrt{2} \Lambda}\left[\frac{c_{1}}{8}\left(1+\beta^{2}\right)+\frac{c_{4}}{2} \beta^{2}-\frac{c_{6}+c_{7} \beta^{2}}{2} i \beta\right], \\
& A_{-0}=A_{0-}=\frac{m_{X}^{3}}{m_{V} \sqrt{2} \Lambda}\left[\frac{c_{1}}{8}\left(1+\beta^{2}\right)+\frac{c_{4}}{2} \beta^{2}+\frac{c_{6}+c_{7} \beta^{2}}{2} i \beta\right], \\
& A_{00}=\frac{m_{X}^{4}}{m_{V}^{2} \sqrt{6} \Lambda}\left[\left(1+\beta^{2}\right)\left(\frac{c_{1}}{8}-\frac{c_{2}}{2} \beta^{2}\right)-\beta^{2}\left(\frac{c_{3}}{2} \beta^{2}-c_{4}\right)\right] .
\end{aligned}
$$

As expected, we find six independent helicity amplitudes for the most general case with two identical massive bosons. Note that only two independent combinations of $c_{5,6,7}$ constructed from $g_{8,9,10}^{(2)}$ enter Eq. (19), and therefore only those combinations are accessible in a measurement.

To describe decays of $X$ into two massless bosons, all helicity amplitudes with a longitudinal polarization in Eq. (19) should be disregarded and $\beta=1$ should be substituted everywhere. The relation between the coefficients $c_{j}$ and couplings $g_{i}^{(2)}$ in this case is found from Eq. (7) where $m_{V}=0$ should be substituted. We note that a peculiar feature emerges as the result of this procedure in the case when the $X$ coupling to massless gauge fields is minimal. The minimal coupling corresponds to $g_{1}^{(2)}=1$ and $g_{j>1}^{(2)}=0$. From Eq. (77), it follows that in that case $c_{2}=-c_{1} / 4=c_{4} / 2$ and all other coefficients are zero. Eq. (19) then implies that, in the case of the minimal coupling of the particle $X$ 
to massless gauge bosons, $A_{++}=A_{--}=0$. Hence, only projections $J_{z}= \pm 2$ of the $X$ spin on the collisions axis are allowed in that case. However, for more general couplings, $A_{++}=A_{--}$amplitudes do not vanish and, therefore, zero projection of the $X$ spin on the collision axes is allowed.

\section{Helicity amplitudes for decays into two fermions}

In this subsection, we write down helicity amplitudes for $X$ coupling to two quarks. We need those helicity amplitudes to describe production of the resonance $X$ in $q \bar{q}$ annihilation. The helicity amplitudes for the spin-zero case read

$$
\begin{aligned}
& A_{++}=\frac{m_{q}}{v} m_{X}\left(\rho_{2}^{(0)}-\beta \rho_{1}^{(0)}\right), \\
& A_{--}=\frac{m_{q}}{v} m_{X}\left(\rho_{2}^{(0)}+\beta \rho_{1}^{(0)}\right) .
\end{aligned}
$$

The helicity amplitudes for the spin-one case read

$$
\begin{aligned}
& A_{++}=-2 m_{q}\left(\rho_{1}^{(1)}+\frac{\beta m_{X}^{2}}{2 \Lambda^{2}}\left(\rho_{4}^{(1)}-\beta \rho_{3}^{(1)}\right)\right) \\
& A_{--}=-2 m_{q}\left(-\rho_{1}^{(1)}+\frac{\beta m_{X}^{2}}{2 \Lambda^{2}}\left(\rho_{4}^{(1)}+\beta \rho_{3}^{(1)}\right)\right), \\
& A_{+-}=\sqrt{2} m_{X}\left(\rho_{1}^{(1)}+\beta \rho_{2}^{(1)}\right) \\
& A_{-+}=-\sqrt{2} m_{X}\left(\rho_{1}^{(1)}-\beta \rho_{2}^{(1)}\right) .
\end{aligned}
$$

The helicity amplitudes for the spin-two case read

$$
\begin{aligned}
& A_{++}=\frac{2 \sqrt{2} m_{q} m_{X} \beta}{\sqrt{3} \Lambda}\left(\rho_{1}^{(2)}+\frac{\beta m_{X}^{2}}{2 \Lambda^{2}}\left(\rho_{4}^{(2)}-\beta \rho_{3}^{(2)}\right)\right), \\
& A_{--}=\frac{2 \sqrt{2} m_{q} m_{X} \beta}{\sqrt{3} \Lambda}\left(-\rho_{1}^{(2)}+\frac{\beta m_{X}^{2}}{2 \Lambda^{2}}\left(\rho_{4}^{(2)}+\beta \rho_{3}^{(2)}\right)\right), \\
& A_{+-}=-\frac{m_{X}^{2} \beta}{\Lambda}\left(\rho_{1}^{(2)}+\beta \rho_{2}^{(2)}\right) \\
& A_{-+}=\frac{m_{X}^{2} \beta}{\Lambda}\left(\rho_{1}^{(2)}-\beta \rho_{2}^{(2)}\right) .
\end{aligned}
$$

\section{ANGULAR DISTRIBUTIONS}

Information about the quantum numbers of $X$ and its couplings to the SM fields can be extracted from the angular distributions. In general, there are five angles that can be studied, see Fig. 1. The two production angles, $\theta^{*}$ and $\Phi_{1}$, are defined relative to the parton collision axis; distributions of those angles depend on the production mechanism. The three helicity angles, $\theta_{1}, \theta_{2}$, and $\Phi$, are sensitive to the structure of the interactions with the resonance decay products, but they do not depend on the production mechanism. These angles are illustrated in Fig. 1 for the process of the type $2 \rightarrow 4$, where the decay chain $X \rightarrow Z_{1} Z_{2} \rightarrow\left(f_{1} \bar{f}_{1}\right)\left(f_{2} \bar{f}_{2}\right)$ is considered and the production mechanism is either $g g \rightarrow X$ or $q \bar{q} \rightarrow X$.

The exact definition of the five angles shown in Fig. 1 is as follows: $\theta^{*}$ is the angle between the parton collision axis $z$ and the $X \rightarrow Z_{1} Z_{2}$ decay axis $z^{\prime}$, both defined in the $X$ rest frame; $\Phi_{1}$ is the angle between the $z z^{\prime}$ plane and the plane of the $Z_{1} \rightarrow\left(f_{1} \bar{f}_{1}\right)$ decay in the $X$ rest frame; $\theta_{i}$ is the angle between the direction of the fermion $f_{i}$ from $Z_{i} \rightarrow\left(f_{i} \bar{f}_{i}\right)$ and the direction opposite the $X$ in the $Z_{i}$ rest frame, where index $i=1,2$ refers to the first or second $Z$ boson; finally, $\Phi$ is the angle between the decay planes of the two $Z$ systems in the $X$ rest frame. The sixth angle $\Phi^{*}$ is the azimuth angle of the $z^{\prime}$ axis with respect to $z$. It can be arbitrarily defined and it does not carry any information about the process. However, $\Phi^{*}$ can be used for bookkeeping purposes to differentiate between the first and second $Z$ in the decay in the case that both have identical decay channels. Discussion of the small possible difference between the $z$ axis and the beam collision axis follows below.

Distributions of the five angles described above allow one to determine the spin of the $X$ boson and measure contributions of the different helicity amplitudes $A_{\lambda_{1} \lambda_{2}}$ in both production and decay. The $X$ boson couplings to 
the SM fields can be deduced from the helicity amplitudes, cf. Eqs. (16 - 222). We therefore proceed to the general expression for the angular distributions in the process $p p \rightarrow X \rightarrow V_{1} V_{2} \rightarrow\left(f_{1} f_{1}^{\prime}\right)\left(f_{2} f_{2}^{\prime}\right)$ and illustrate the general formula by considering a number of specific examples. We use the helicity formalism as described in Refs. 26, 27]; for recent examples of applications of the helicity formalism, see Refs. [8, 9, 11, 28 31]. Our results are consistent with those references but are, typically, more general.

Let us first consider the $1 \rightarrow 2$ decay process. The helicity amplitudes are defined through a matrix element of the scattering matrix between states with definite projections of angular momenta on a chosen quantization axis. Hence,

$$
\left\langle\Omega, \lambda_{1}, \lambda_{2}|S| J m\right\rangle=\sqrt{\frac{(2 J+1)}{4 \pi}} D_{m, \lambda_{1}-\lambda_{2}}^{J *}(\Omega) A_{\lambda_{1} \lambda_{2}},
$$

where $\Omega$ describes the polar and the azimuthal angles of one of the final state particles and $D$ denotes the corresponding Wigner function. Viewing the collision process of two partons $a$ and $b, a b \rightarrow X \rightarrow V_{1} V_{2} \rightarrow\left(f_{1} f_{1}^{\prime}\right)\left(f_{2} f_{2}^{\prime}\right)$, as a sequence of $1 \leftrightarrow 2$ elementary processes, we describe it by the following formula

$$
\begin{aligned}
& A_{a b}\left(p_{a}, p_{b} ; \chi_{1}, \chi_{2}, m, \lambda_{1}, \lambda_{2}, \mu_{1}, \mu_{2}, \tau_{1}, \tau_{2} ; \Omega^{*}, \Omega, \Omega^{\prime}, \Omega^{\prime \prime}\right)=\frac{(2 J+1)}{4 \pi} \sqrt{\frac{\left(2 s_{1}+1\right)}{4 \pi} \frac{\left(2 s_{2}+1\right)}{4 \pi}} \\
& \times D_{\chi_{1}-\chi_{2}, m}^{J *}\left(\Omega^{*}\right) B_{\chi_{1} \chi_{2}} \times D_{m, \lambda_{1}-\lambda_{2}}^{J *}(\Omega) A_{\lambda_{1} \lambda_{2}} \times D_{\lambda_{1}, \mu_{1}-\mu_{2}}^{s_{1} *}\left(\Omega^{\prime}\right) T\left(\mu_{1}, \mu_{2}\right) \times D_{\lambda_{2}, \tau_{1}-\tau_{2}}^{s_{2} *}\left(\Omega^{\prime \prime}\right) W\left(\tau_{1}, \tau_{2}\right) .
\end{aligned}
$$

Here the $A_{\lambda_{1} \lambda_{2}}$ and $B_{\chi_{1} \chi_{2}}$ amplitudes correspond to the $X$ decay and production processes, respectively; $T\left(\mu_{1}, \mu_{2}\right)$ and $W\left(\tau_{1}, \tau_{2}\right)$ describe the decays of the first and the second $X$ daughter to pairs of fermions; $\lambda_{1,2}$ are the helicities of the two $X$ daughters; $\chi_{1,2}$ are the helicities of two gluons or quarks in the initial state; $\mu_{1,2}$ and $\tau_{1,2}$ are the helicities of fermions in the $Z$ decays; and $m$ denotes the helicity of $X$. We keep the notation general enough with $J$ denoting the spin of the $X$ boson and $s_{1,2}$ referring to the spins of its decay products, e.g. the $Z$ bosons. We choose the convention where $\Omega=(0,0,0)$, which by conservation of angular momentum effectively sets $m=\lambda_{1}-\lambda_{2}$, $\Omega^{*}=\left(\Phi_{1}, \theta^{*},-\Phi_{1}\right), \Omega^{\prime}=\left(0, \theta_{1}, 0\right)$ and $\Omega^{\prime \prime}=\left(\Phi, \theta_{2},-\Phi\right)$. Summing over the helicities of all particles, we obtain the differential cross-section for the process $a b \rightarrow X \rightarrow V_{1} V_{2} \rightarrow\left(f_{1} f_{1}^{\prime}\right)\left(f_{2} f_{2}^{\prime}\right)$ as

$$
\frac{d \sigma_{a b}\left(p_{a}, p_{b}, \theta^{*}, \Phi_{1}, \theta_{1}, \theta_{2}, \Phi\right)}{\mathrm{d} Y_{X} d \cos \theta^{*} d \Phi_{1} d \cos \theta_{1} d \cos \theta_{2} d \Phi}=\mathcal{N}_{a b} \delta\left(s_{a b}-m_{X}^{2}\right) \delta\left(Y_{a b}-Y_{X}\right) \sum_{\{\chi, \mu, \tau\}}\left|\sum_{\{\lambda, m\}} A_{a b}\left(p_{a}, p_{b} ;\{\chi, \lambda ; m, \mu, \tau\} ;\{\Omega\}\right)\right|^{2},
$$

where $s_{a b}$ is the partonic center-of-mass energy squared, $Y_{a b}$ and $Y_{X}$ are the rapidities of the colliding partons $a b$ and the resonance $X$, and $\mathcal{N}_{a b}$ is the normalization factor

$$
\mathcal{N}_{a b}=\frac{\pi \beta_{Z}}{8 m_{X}^{3} \Gamma_{X} c_{a b}\left(32 \pi^{2}\right)^{3}\left(2 m_{Z} \Gamma_{Z}\right)^{2}},
$$

with $\beta_{Z}=\sqrt{1-4 m_{Z}^{2} / m_{X}^{2}}, c_{q \bar{q}}=3, c_{g g}=8$, and $\Gamma_{X, Z}$ being the decay widths of the resonance $X$ and the $Z$ boson, respectively. The relevant differential cross-section for hadron collisions is obtained by convoluting parton cross-sections with parton distribution functions

$$
\frac{d \sigma_{p p}\left(\theta^{*}, \Phi_{1}, \theta_{1}, \theta_{2}, \Phi\right)}{d \cos \theta^{*} d \Phi_{1} d \cos \theta_{1} d \cos \theta_{2} d \Phi}=\left.\sum_{a b} \int \mathrm{d} Y_{X} \mathrm{~d} x_{1} \mathrm{~d} x_{2} \tilde{f}_{a}\left(x_{1}\right) \tilde{f}_{b}\left(x_{2}\right) \frac{d \sigma_{a b}\left(x_{1} p_{1}, x_{2} p_{2}, \theta^{*}, \Phi_{1}, \theta_{1}, \theta_{2}, \Phi\right)}{\mathrm{d} Y_{X} d \cos \theta^{*} d \Phi_{1} d \cos \theta_{1} d \cos \theta_{2} d \Phi}\right|_{Y_{a b}=\frac{1}{2} \ln \frac{x_{1}}{x_{2}}},
$$

where $p_{1,2}$ are the momenta of the two protons. Note that the angles $\cos \theta^{*}, \Phi_{1}, \cos \theta_{1}, \cos \theta_{2}, \Phi$ are defined in the $X$ rest frame and are not affected by the integrations over $x_{1}, x_{2}$, and $Y_{X}$. Convolution with the parton distribution functions results in the rapidity distribution of the $X$ boson and may affect angular distributions of decay products on an event-by-event basis due to the detector acceptance, discussed later. However, it does not affect angular distributions measured with the ideal detector since in this case, integrations over $x_{1}, x_{2}$, and $Y_{X}$ in Eq. (27) factorize from all angular dependences. As the result, we may rewrite Eq. (27) in the form of the angular distribution of the decay products of the polarized particle $X$

$$
\frac{1}{\sigma_{p p, \text { tot }}} \frac{d \sigma_{p p}}{d \cos \theta^{*} d \Phi_{1} d \cos \theta_{1} d \cos \theta_{2} d \Phi}=\frac{1}{\Gamma_{X}} \frac{\mathrm{d} \Gamma_{X}}{d \cos \theta^{*} d \Phi_{1} d \cos \theta_{1} d \cos \theta_{2} d \Phi} .
$$

Since we are interested in the normalized distributions, many normalization factors, as in Eq. (26), drop out. However, ratios of amplitudes squared $\left|A_{a b}\right|^{2}$ and ratios of partonic luminosities for different production channels appear in the normalized angular distributions and contribute to the degree of the polarization of the particle X. Note that if the $X$ particle can only be produced in a single partonic channel, all dependence on the partonic luminosities cancels out. 
This is relevant for both a spin-zero and spin-one $X$ which are produced in $a b=g g$ and $q \bar{q}$ collisions, respectively. For a spin-two $X$ particle, the two parton channels contribute and their relative partonic luminosities affect the relative fraction of spin-projection-one polarization as we note below. Apart from this relative normalization, which is incorporated into notation discussed below, we can derive angular distributions using the sum of $\left|A_{a b}\right|^{2}$ over the helicity states, as indicated in Eq. (25), for each parton channel $a b$ independently.

To define easy-to-measure parameters related to the helicity amplitudes, we consider the case of a spin-two resonance as an example. The decay of a spin-two particle to two vector bosons is characterized by nine complex helicity amplitudes $A_{\lambda_{1} \lambda_{2}}$. When vector bosons in the final state are identical, only six helicity amplitudes remain independent. Six complex amplitudes are parameterized by twelve real numbers. However, since we are interested in the normalized angular distribution, two of them, the normalization and the overall phase, are unobservable. Hence, angular distributions of the spin-two particle decay to two identical vector bosons can be parameterized by ten real parameters. We can choose them to be

$$
\begin{aligned}
f_{\lambda_{1} \lambda_{2}} & =\left|A_{\lambda_{1} \lambda_{2}}\right|^{2} / \sum_{k, l= \pm, 0}\left|A_{k l}\right|^{2}, \\
\phi_{\lambda_{1} \lambda_{2}} & =\arg \left(A_{\lambda_{1} \lambda_{2}} / A_{00}\right),
\end{aligned}
$$

with $\left(\lambda_{1}, \lambda_{2}\right)=(++),(--),(+-),(+0),(0-)$. The remaining (dependent) amplitude parameters $f_{k l}$ and $\phi_{k l}$ can be expressed either using Eq. (14) or the relationship

$$
f_{00}=1-\sum_{k, l= \pm, 0} f_{k l}, \quad \phi_{00}=0 .
$$

For the $X$ decays to massless vector bosons or if its spin is less than two, the number of independent non-vanishing parameters is further reduced. Our notation for those (simpler) cases are similar to what is described above. For example, the spin-zero case is parameterized with four real parameters for $\left(\lambda_{1}, \lambda_{2}\right)=(++),(--)$, and the spin-one case with two real parameters for $\left(\lambda_{1}, \lambda_{2}\right)=(+0)$ after re-defining the phase convention with respect to the $A_{0-}$ amplitude.

Equivalent parameters can be defined for the helicity amplitudes $B_{\chi_{1} \chi_{2}}$ describing the production mechanism. However, not all parameters would enter the angular distributions. We choose the three real parameters $f_{z 0}, f_{z 1}$, and $f_{z 2}$ to describe the fraction of spin-two $X$ resonance production with spin $z$-projections $0, \pm 1$, and \pm 2 , respectively, where $f_{z 0}+f_{z 1}+f_{z 2} \equiv 1$, leaving only two independent parameters. The fractions $f_{z 0}$ or $f_{z 2}$ arise from the gluon fusion mechanism with amplitudes $B_{++}$and $B_{--}$or $B_{+-}$and $B_{-+}$in Eq. (19), respectively, where we now use the $B_{\chi_{1} \chi_{2}}$ notation in place of $A_{\chi_{1} \chi_{2}}$ to distinguish the process of production from decay. The fraction $f_{z 1}$ originates from $q \bar{q}$ annihilation with amplitudes $B_{+-}$and $B_{-+}$in Eq. (22). Note that because we assume that chirality is a good quantum number, only \pm 1 spin projections on the $z$ axis are possible in the annihilation of massless quarks. On the other hand, both $J_{z}= \pm 2$ and $J_{z}=0$ spin projections of a spin-two $X$ are possible in gluon fusion. The latter possibility is often ignored in the literature because, accidentally, this contribution vanishes in the case of the minimal coupling of the spin-two particle to massless gauge bosons. Finally we note that, since $f_{z 1}$ and $f_{z 0}, f_{z 2}$ are produced by different partonic initial states, those quantities are proportional to corresponding partonic luminosities, in addition to the production helicity amplitudes $B_{\chi_{1} \chi_{2}}$. Below we discuss simplified versions of the general angular distribution which are obtained upon integrating over some of the angles in Eq. (27).

\section{A. Distributions of production angles}

We consider the $g g$ and $q \bar{q}$ production of an exotic resonance $X$ and its subsequent decay $X \rightarrow Z_{1} Z_{2} \rightarrow\left(f_{1} \bar{f}_{1}\right)\left(f_{2} \bar{f}_{2}\right)$. Angular distributions for other decay channels such as $X \rightarrow \gamma \gamma, X \rightarrow g g$, and $X \rightarrow f \bar{f}$ are given in the appendix.

The production angle $\theta^{*}$ is shown in Fig. 1 for an $X$ decaying to two $Z$ bosons, as an example. It is defined as the angle between the parton collision axis $z$ and the $X$ decay axis in the $X$ rest frame. Determination of the $z$ axis requires care since it may differ somewhat from the beam collision axis which we discuss below. The distribution of the angle $\theta^{*}$ is the only angular observable that contains information about the spin and parity properties of the particle $X$, unless decay chains of the $X$ daughters are analyzed. The latter is possible in the decay to two massive vector bosons $Z Z$ or $W^{+} W^{-}$for certain cases, but is challenging or practically impossible in other cases. The production angle distributions have been extensively studied in the literature [13 18]. However, for the spin-two resonance, we point to several modifications of the standard formulas due to generally ignored $J_{z}=0$ projection on the collision axis.

Transverse momentum of the $X$ particle introduces uncertainties in the production angle determination. The Collins-Soper frame [32] is designed to minimize the effect of $X$ transverse momentum by placing the $z$ axis half 
way between axes of two beams in the $X$ rest frame. Note that if $X$ has transverse momentum, the two beams are no longer collinear in the $X$ rest frame. The uncertainty introduced in the $\theta^{*}$ measurement by the non-vanishing transverse momentum of the $X$ particle is expected to be relatively small [17], at least when compared to the statistical uncertainties in early LHC measurements. With a larger number of events becoming available later, one can, on an event-by-event basis, find and disregard events where $X$ recoils against hadronic jets with a large transverse momentum.

Note that there is an ambiguity in the direction of the $z$ axis and one cannot distinguish $\theta^{*}$ from $\left(\pi-\theta^{*}\right)$ when two identical particles are involved in either the production or decay of the $X$ particle. In particular, this happens if $X$ is produced in gluon fusion or when $X$ decays to two identical bosons, such as $Z Z, g g$, or $\gamma \gamma$. In those cases, terms with an odd power in $\cos \theta^{*}$ drop out from the angular distributions and we do not consider them further. Only when $X$ is produced in $q \bar{q}$ annihilation and decays into a particle-antiparticle pair, such as $W^{+} W^{-}$or $l^{+} l^{-}$, can one try to deduce the $\cos \theta^{*} \operatorname{sign}$.

The case of a spin-zero particle is very simple. Since no spin correlations are involved, the production angles distributions are flat. However, determination of these angles is still relevant for the analysis of a spin-zero particle because it is needed to discriminate against backgrounds or resonances with non-zero spin. Normalized distributions of production angles for spin-one and spin-two resonances that decay as $X \rightarrow Z Z \rightarrow\left(f_{1} \bar{f}_{1}\right)\left(f_{2} \bar{f}_{2}\right)$ can be written as follows

$$
\frac{32 \pi d \Gamma_{J=1}}{3 \Gamma d \cos \theta^{*} d \Phi_{1}}=2\left(1+\cos ^{2} \theta^{*}\right)-\sqrt{2 f_{+0}\left(1-2 f_{+0}\right)} \sin ^{2} \theta^{*} \cos \left(2 \Phi_{1}-\left[\phi_{+0}-\phi_{0-}\right]\right)
$$

$$
\begin{aligned}
& \frac{32 \pi d \Gamma_{J=2}}{5 \Gamma d \cos \theta^{*} d \Phi_{1}}=\left(2-2 f_{z 1}+f_{z 2}\right)-6\left(2-4 f_{z 1}-f_{z 2}\right) \cos ^{2} \theta^{*}+3\left(6-10 f_{z 1}-5 f_{z 2}\right) \cos ^{4} \theta^{*} \\
& +f_{+-}\left\{\left(2+2 f_{z 1}-7 f_{z 2}\right)+6\left(2-6 f_{z 1}+f_{z 2}\right) \cos ^{2} \theta^{*}-5\left(6-10 f_{z 1}-5 f_{z 2}\right) \cos ^{4} \theta^{*}\right\} \\
& -2\left(f_{+0}+f_{0-}\right)\left\{\left(2-4 f_{z 1}-f_{z 2}\right)-6\left(4-7 f_{z 1}-3 f_{z 2}\right) \cos ^{2} \theta^{*}+5\left(6-10 f_{z 1}-5 f_{z 2}\right) \cos ^{4} \theta^{*}\right\} \\
& -2 \sqrt{f_{+0} f_{0-}}\left\{\left(f_{z 1}-f_{z 2}\right)+\left(6-10 f_{z 1}-5 f_{z 2}\right) \cos ^{2} \theta^{*}\right\} \sin ^{2} \theta^{*} \cos \left(2 \Phi_{1}+\phi_{+0}-\phi_{0-}\right) \\
& -\frac{1}{2}\left\{\left(2-2 f_{z 1}-3 f_{z 2}\right)-\left(6-10 f_{z 1}-5 f_{z 2}\right) \cos ^{2} \theta^{*}\right\} \sin ^{2} \theta^{*} \\
& \quad \times\left[\sqrt{6 f_{++} f_{+-}} \cos \left(2 \Phi_{1}-\phi_{++}+\phi_{+-}\right)+\sqrt{6 f_{--} f_{+-}} \cos \left(2 \Phi_{1}+\phi_{--}-\phi_{+-}\right)\right] \\
& +\frac{3 \pi R_{1}}{4}\left\{\left(2-4 f_{z 1}-f_{z 2}\right)-\left(6-10 f_{z 1}-5 f_{z 2}\right) \cos ^{2} \theta^{*}\right\} \cos \theta^{*} \sin \theta^{*} \\
& \quad \times\left[\sqrt{3 f_{+0}\left(1-f_{++}-f_{--}-2 f_{+-}-2 f_{+0}-2 f_{0-}\right)} \cos \left(\Phi_{1}-\phi_{+0}\right)+\sqrt{3 f_{+0} f_{++}} \cos \left(\Phi_{1}+\phi_{+0}-\phi_{++}\right)\right. \\
& \left.\quad-\sqrt{3 f_{0-}\left(1-f_{++}-f_{--}-2 f_{+-}-2 f_{+0}-2 f_{0-}\right)} \cos \left(\Phi_{1}+\phi_{0-}\right)-\sqrt{3 f_{0-} f_{--}} \cos \left(\Phi_{1}-\phi_{0-}+\phi_{--}\right)\right] \\
& +\frac{3 \pi R_{1}}{8}\left\{\left(6-6 f_{z 1}-9 f_{z 2}\right)-\left(6-10 f_{z 1}-5 f_{z 2}\right) \cos ^{2} \theta^{*}\right\} \cos \theta^{*} \sin \theta^{*} \\
& \quad \times\left[\sqrt{2 f_{+-} f_{+0}} \cos \left(\Phi_{1}+\phi_{+-}-\phi_{+0}\right)-\sqrt{2 f_{+-} f_{0-}} \cos \left(\Phi_{1}-\phi_{+-}+\phi_{0-}\right)\right] .
\end{aligned}
$$

Note that the right hand side of Eq. (33) is written as a linear combination of terms of the form $\left(\alpha_{1}+\alpha_{2} f_{z 1}+\alpha_{3} f_{z 2}\right)$, multiplied by other parameters. It is peculiar that in all such terms, except in the very first, $\theta^{*}-$ and $\Phi_{1}$-independent one, there is a relationship between the $\alpha$-coefficients $\alpha_{1}=-0.4 \times\left(\alpha_{2}+\alpha_{3}\right)$. This means that if we choose $f_{z 1}=$ $f_{z 2}=0.4$ in Eq. (33), the production angle distribution for the spin-two particle becomes flat! To understand this, note that those helicity fractions imply production of an unpolarized $X$-boson in which case the production angle distribution must be constant.

It is interesting to point out in this regard that observation of the flat production angle distribution in a twobody decay $X \rightarrow P_{1} P_{2}$ does not mean that the $X$ 's spin is zero. In fact, $X$ can have any spin $J$ but, for the flat distribution, it must be produced unpolarized. In turn, this implies that all helicity fractions must be equal $2 f_{z 0}=f_{z 1}=f_{z 2}=\ldots f_{z J}$. In general, each helicity fraction $f_{z m}$ is a sum of many terms

$$
f_{z m} \sim \sum_{a b}\left|g_{a b \rightarrow X, m}\right|^{2} \tilde{f}_{a} \tilde{f}_{b}
$$

where $a, b$ are the two partons whose collision produces the resonance $X, g_{a b \rightarrow X, m}$ are the couplings of the two partons to the resonance $X$ in the helicity state $m$ and $\tilde{f}_{a, b}$ are the parton distribution functions. It follows that the equality 
of all helicity fractions requires an unnatural tuning between the coupling constants and the parton distribution functions. For example, a spin-one resonance can only be single-produced in $q \bar{q}$ collisions and, in general, there are two helicity fractions $f_{z 0}$ and $f_{z 1}$. Since, as follows from Eq. (21), $f_{z 0}$ is proportional to the quark mass squared, it is very unnatural to expect unpolarized production in the $J=1$ case. For an $X$ particle of spin two, unpolarized production requires tuning of the coupling constants and parton distributions since gluon collisions are responsible for the $f_{z 0}$ and $f_{z 2}$ helicity fractions and $q \bar{q}$ collisions - for the $f_{z 1}$ helicity fraction. It is interesting that for a fixed mass $X$ resonance, such tuning can only be argued for a particular energy of the hadron collider; changing the collider energy, at least as a matter of principle, will clearly destroy the tuning and, if $J>0$, will turn flat $\cos \theta^{*}$ distributions into non-flat. While the above discussion shows that unpolarized production of the resonance with non-vanishing spin requires a high degree of tuning between coupling constants and parton distribution functions, we emphasize that, as a matter of principle, observation of a flat production angle distribution does not immediately imply that the spin of the resonance is zero. As we discuss next, the analysis of helicity angle distributions helps in distinguishing the different spin scenarios.

\section{B. Distributions of helicity angles}

It follows from Eq. (25) that the most general angular distribution depends on five angles. Such a distribution contains information about production of the resonance $X$ and its decay into $Z Z \rightarrow\left(f_{1} \bar{f}_{1}\right)\left(f_{2} \bar{f}_{2}\right)$. In this section, we restrict the presentation to angular distributions which are averaged over the production angle $\Phi_{1}$. The most general angular distributions are given in the appendix. To obtain distributions differential in the three helicity angles, one can easily integrate over the $\cos \theta^{*}$ in the formulas below. In a similar manner, it is easy to obtain one-dimensional projection of any of the five angles that describe the decay. In this subsection, we present those distributions for spin-zero, spin-one, and spin-two resonances that decay as $X \rightarrow Z Z \rightarrow\left(f_{1} \bar{f}_{1}\right)\left(f_{2} \bar{f}_{2}\right)$. Because $Z$ decays are involved, distributions depend on the parameters $R_{1,2}=2 r_{1,2} /\left(1+r_{1,2}^{2}\right)$, where $r_{1,2}$ is the ratio of axial to vector couplings of the fermions $f_{1,2}$. Specifically, $r=c_{A} / c_{V}=t_{3 L} /\left(t_{3 L}-2 q \sin ^{2} \theta_{W}\right)$, where $q$ is the fermion charge and $t_{3 L}$ is its weak isospin. In this paper we mostly consider $Z$ decays to charged leptons. For them, $q=-1, t_{3 L}=-1 / 2$, and $R \simeq 0.15$. Note that if the first $Z$ decays to charged leptons and the second $Z$ decays to quark and antiquark jets, one needs to average the value of $R_{2}$ over contributing quark flavors since $R$ is different for up and down quarks, $R_{\text {up }} \simeq 0.67, R_{\text {down }} \simeq 0.94$. However, if the quark and antiquark jets cannot be distinguished in the $Z$ decay, this is equivalent to $R_{2}=0$ in the measured angular distributions.

The angular distribution for a spin-zero resonance is independent of the production angles. It depends on four free parameters and reads

$$
\begin{aligned}
\frac{128 \pi d \Gamma \Gamma_{J=0}}{9 \Gamma d \cos \theta_{1} d \cos \theta_{2} d \Phi}= & 4\left(1-f_{++}-f_{--}\right) \sin ^{2} \theta_{1} \sin ^{2} \theta_{2} \\
& +\left(f_{++}+f_{--}\right)\left(\left(1+\cos ^{2} \theta_{1}\right)\left(1+\cos ^{2} \theta_{2}\right)+4 R_{1} R_{2} \cos \theta_{1} \cos \theta_{2}\right) \\
& -2\left(f_{++}-f_{--}\right)\left(R_{1} \cos \theta_{1}\left(1+\cos ^{2} \theta_{2}\right)+R_{2}\left(1+\cos ^{2} \theta_{1}\right) \cos \theta_{2}\right) \\
& +4 \sqrt{f_{++}\left(1-f_{++}-f_{--}\right)}\left(R_{1}-\cos \theta_{1}\right) \sin \theta_{1}\left(R_{2}-\cos \theta_{2}\right) \sin \theta_{2} \cos \left(\Phi+\phi_{++}\right) \\
& +4 \sqrt{f_{--}\left(1-f_{++}-f_{--}\right)}\left(R_{1}+\cos \theta_{1}\right) \sin \theta_{1}\left(R_{2}+\cos \theta_{2}\right) \sin \theta_{2} \cos \left(\Phi-\phi_{--}\right) \\
& +2 \sqrt{f_{++} f_{--}} \sin ^{2} \theta_{1} \sin ^{2} \theta_{2} \cos \left(2 \Phi+\phi_{++}-\phi_{--}\right) .
\end{aligned}
$$

We point out that non-zero values of $R_{i}$ are reflected in preferential directions of fermions in $Z$ decays, see e.g. terms $R_{1} \cos \theta_{1}$ and $R_{2} \cos \theta_{2}$ that are present in Eq. (35) if parity is violated with $f_{++} \neq f_{--}$. Equation (35) is the most general angular distribution of the decay of a spin-zero particle and, as such, generalizes many similar results presented in the literature.

We note, however, that in specific cases the number of parameters in Eq. (35) can be reduced. For example, considering the tree-level coupling of the SM Higgs boson to two $Z$ bosons, we find the relation between three helicity amplitudes to be $A_{++}=A_{--}=-A_{00} / \gamma$, where $\gamma$ is defined in Eq. (17). This leads to

$$
f_{++}=f_{--}=\frac{1}{\gamma^{2}+2}, \quad \phi_{++}=\phi_{--}=\pi .
$$

It is easy to account for changes in $H \rightarrow Z Z \rightarrow 4 l$ angular distributions caused by the radiative corrections in the SM, if $a_{2}^{\mathrm{SM}}$ and $a_{1}^{\mathrm{SM}}$ are known. Similarly, for a parity-conserving interaction of a $J^{P}=0^{-}$particle, one has $f_{++}=f_{--}=1 / 2$ and $\phi_{++}-\phi_{--}=\pi$. 
The angular distribution for a spin-one particle decaying to $X \rightarrow Z Z$ is determined by two parameters; we choose them to be $f_{+0}$ and $\left[\phi_{+0}-\phi_{0-}\right]=\arg \left(A_{+0} / A_{0-}\right)$. The angular distribution reads

$$
\begin{aligned}
\frac{512 \pi d \Gamma_{J=1}}{27 \Gamma d \cos \theta^{*} d \cos \theta_{1} d \cos \theta_{2} d \Phi}= & \left\{1+\cos ^{2} \theta^{*}\right\} \times\left\{1-\cos ^{2} \theta_{1} \cos ^{2} \theta_{2}+\left(1-4 f_{+0}\right)\left(R_{1} \cos \theta_{1} \sin ^{2} \theta_{2}+R_{2} \sin ^{2} \theta_{1} \cos \theta_{2}\right)\right. \\
& \left.+\sqrt{8 f_{+0}\left(1-2 f_{+0}\right)} \sin \theta_{1} \sin \theta_{2}\left(R_{1} R_{2}-\cos \theta_{1} \cos \theta_{2}\right) \cos \left(\Phi+\left[\phi_{+0}-\phi_{0-}\right]\right)\right\}
\end{aligned}
$$

This distribution differs from a recent result presented in Ref. [9]. The origin of the difference is the reality of the two effective couplings $g_{1,2}^{(1)}$ assumed in that reference. Indeed, if the two couplings are real then, independent of their actual values, $f_{+0}=1 / 4$, as follows from Eq. (18). Then, the terms in Eq. (37) that are linear in $R_{i}$ do not contribute. In the more general case of two complex couplings, the preferential polarization of the $Z$ boson appears and gets reflected in the preferential direction of, say, the negatively charged lepton in $Z$-decays. We also note that the angular dependence in Eq. (37) is a product of the production angle distribution and the distribution of three helicity angles.

Finally, we consider a spin-two resonance. The normalized angular distribution is

$$
\begin{aligned}
& \frac{2048 \pi d \Gamma_{J=2}}{45 \Gamma d \cos \theta^{*} d \cos \theta_{1} d \cos \theta_{2} d \Phi}=\left\{\left(2-2 f_{z 1}+f_{z 2}\right)-6\left(2-4 f_{z 1}-f_{z 2}\right) \cos ^{2} \theta^{*}+3\left(6-10 f_{z 1}-5 f_{z 2}\right) \cos ^{4} \theta^{*}\right\} \\
& \times\left\{4\left(1-f_{++}-f_{--}-2 f_{+-}-2 f_{+0}-2 f_{0-}\right) \sin ^{2} \theta_{1} \sin ^{2} \theta_{2}\right. \\
& \quad+\left(f_{++}+f_{--}\right)\left(\left(1+\cos ^{2} \theta_{1}\right)\left(1+\cos ^{2} \theta_{2}\right)+4 R_{1} R_{2} \cos \theta_{1} \cos \theta_{2}\right) \\
& \quad-2\left(f_{++}-f_{--}\right)\left(R_{1} \cos \theta_{1}\left(1+\cos ^{2} \theta_{2}\right)+R_{2}\left(1+\cos ^{2} \theta_{1}\right) \cos \theta_{2}\right) \\
& \quad+4 \sqrt{f_{++}\left(1-f_{++}-f_{--}-2 f_{+-}-2 f_{+0}-2 f_{0-}\right)}\left(R_{1}-\cos \theta_{1}\right) \sin \theta_{1}\left(R_{2}-\cos \theta_{2}\right) \sin \theta_{2} \cos \left(\Phi+\phi_{++}\right) \\
& \quad+4 \sqrt{f_{--}\left(1-f_{++}-f_{--}-2 f_{+-}-2 f_{+0}-2 f_{0-}\right)}\left(R_{1}+\cos \theta_{1}\right) \sin \theta_{1}\left(R_{2}+\cos \theta_{2}\right) \sin \theta_{2} \cos \left(\Phi-\phi_{--}\right) \\
& \left.\quad+2 \sqrt{f_{++} f_{--}} \sin ^{2} \theta_{1} \sin ^{2} \theta_{2} \cos \left(2 \Phi+\phi_{++}-\phi_{--}\right)\right\} \\
& +8\left\{\left(f_{z 1}+f_{z 2}\right)+3\left(2-3 f_{z 1}-2 f_{z 2}\right) \cos ^{2} \theta^{*}-\left(6-10 f_{z 1}-5 f_{z 2}\right) \cos ^{4} \theta^{*}\right\} \\
& \quad \times\left\{\left(f_{+0}+f_{0-}\right)\left(1-\cos ^{2} \theta_{1} \cos ^{2} \theta_{2}\right)-\left(f_{+0}-f_{0-}\right)\left(R_{1} \cos \theta_{1} \sin ^{2} \theta_{2}+R_{2} \sin ^{2} \theta_{1} \cos \theta_{2}\right)\right. \\
& \left.\quad+2 \sqrt{f_{+0} f_{0-}} \sin \theta_{1} \sin \theta_{2}\left(R_{1} R_{2}-\cos _{1} \cos \theta_{2}\right) \cos \left(\Phi+\left[\phi_{+0}-\phi_{0-}\right]\right)\right\} \\
& +\left\{\left(6-2 f_{z 1}-5 f_{z 2}\right)-6\left(2-2 f_{z 1}-3 f_{z 2}\right) \cos ^{2} \theta^{*}+\left(6-10 f_{z 1}-5 f_{z 2}\right) \cos ^{4} \theta^{*}\right\} \\
& \quad \times f_{+-}\left\{\left(1+\cos ^{2} \theta_{1}\right)\left(1+\cos ^{2} \theta_{2}\right)-4 R_{1} R_{2} \cos \theta_{1} \cos \theta_{2}\right\}
\end{aligned}
$$

where the dependent parameter $f_{00}$ is expressed as $\left(1-f_{++}-f_{--}-2 f_{+-}-2 f_{+0}-2 f_{0-}\right)$.

There are two ways to obtain an angular distribution in the three helicity angles from Eq. (38). One way is to integrate over the $\cos \theta^{*}$ in Eq. (38); note that Eq. (38) is written in such a way that the dependence on $\cos \theta^{*}$ in each term is factored out. Upon integrating over $\cos \theta^{*}$, the dependence on $f_{z 1}$ and $f_{z 2}$ must disappear since the distribution over helicity angles is independent of the production mechanism. We note that this independence suggests an alternative way to obtain the distribution of the helicity angles from Eq. (38). Imagine that $X$ is produced unpolarized, which amounts to setting $f_{z 1}=f_{z 2}=0.4$ in Eq. (38). In this case coefficients of all $\cos \theta^{*}$-dependent terms in Eq. (38) vanish and integration over that angle becomes trivial.

Even if $X$ is produced unpolarized, it is still possible to separate spin hypotheses by analysis of the helicity angles. The opposite situation is also possible when helicity angles provide no separation while productions angle distributions are different. In fact, joint analysis of production and helicity angular distributions is the most efficient way to separate different hypotheses. However, there is a special case of the helicity fractions $f_{+-}=f_{+0}=f_{0-}=0$ and production polarization $f_{z 1}=f_{z 2}=0.4$ where the angular distributions in Eqs. (35) and (38) become identical. The situation $f_{+-}=f_{+0}=f_{0-}=0$ is realized when only terms with $c_{2}, c_{3}$, and $c_{5}$ contribute to helicity amplitudes in Eq. (6), which becomes equivalent to Eq. (2). This is possible, though very unnatural, situation where the $A_{+-}$ amplitude contributes in production but vanishes in the decay mechanism. However, this possibility cannot be ruled out experimentally unless different spin projections in the production are probed.

It is a general feature of Eq. (38) written for spin-two decays that it includes both Eqs. (35) and (37) written for spin-zero and spin-one, respectively. Note that Eq. (38) factorizes into the product of a function of $\cos \theta^{*}$ and a function of three helicity angles under the following three conditions. In the case $f_{+-}=f_{+0}=f_{0-}=0$, the helicity 
angular distribution is given by Eq. (35) and the production angle distribution appears in Eq. (33). This trivially reduces to the case of unpolarized production discussed above. In the other case $f_{0-}+f_{+0}=1 / 2$, and therefore the other $f_{k l}=0$, the helicity angular distribution is given by Eq. (37) and the production angle distribution can be read from Eq. (33). In this case it happens that, under the condition $f_{z 1}=0.6$ and $f_{z 2}=0$, the function of the production angles is proportional to $\left(1+\cos ^{2} \theta^{*}\right)$ and Eqs. (37) and (38) become identical. However, this is only a coincidence and the full angular distributions which also include angle $\Phi_{1}$ do not match, as for example Eqs. (32) and (33) are not identical in this case. Finally, the last term in Eq. (38) corresponds to the non-zero $A_{+-}=A_{-+}$amplitudes and is unique to the spin-two case, though its helicity angular distribution is very similar to the other transverse term which remains in the case $f_{++}=f_{--}=1 / 2$.

\section{MONTE CARLO SIMULATION}

We have written a Monte Carlo program to simulate the production and decay of spin-zero, spin-one, and spin-two resonances in hadron-hadron collisions, including all spin correlations, in the processes $g g(q \bar{q}) \rightarrow X \rightarrow Z Z \rightarrow 4 l$. A special feature of our program, that distinguishes it from other recent implementations [33], is that it includes the most general couplings of the $X$ particle to gluons and fermions in production and to $Z$ bosons in decay, as explained in the previous sections. Extension to other final states, including hadronic $Z$ decays, is straightforward.

The spin-zero resonance is produced in collisions of gluons, and the spin-one resonance is produced in $q \bar{q}$ collisions. For the spin-two resonance, both partonic channels are included in the simulation. Since, in the general case, the relative strength of $g g X$ and $q \bar{q} X$ couplings is not known, the program allows the request that the spin-two $X$ particle is produced with fixed relative frequency in $q \bar{q}$ and $g g$ collisions. We note that both the resonance $X$ and the two $Z$ bosons are considered to be on-shell in our program; all the off-shell effects are neglected. The narrow width approximation is a reasonable assumption for the $Z$ bosons, but it is not possible to say a priori if this also is a good approximation for the resonance $X$. The applicability of the narrow width approximation for the particle $X$ is the assumption built into our program, but it is relatively straightforward to remove this restriction in the future.

The program can output both weighted and unweighted events, depending on the requested mode of operation. Weighted events are typically used for fast calculations of simple one-dimensional distributions and for debugging purposes. Unweighted events, on the other hand, are used to interface the results of our program to programs that simulate realistic detector response.

We now turn to the discussion of how backgrounds of the resonance $X$ for four-lepton production in hadron collisions are simulated. Such backgrounds arise from $Z(\gamma) Z(\gamma), Z b \bar{b}, t \bar{t}, W^{+} W^{-} b \bar{b}, W W Z, t \bar{t} Z$, $4 b$ etc, where we assume that $b$-quarks decay semileptonically. Backgrounds that involve $b$-decays into leptons can be controlled by requiring that leptons are isolated. Other backgrounds can be strongly suppressed by requiring that the invariant masses of lepton pairs are close to the $Z$-boson mass, that there is no missing energy in the event, and that the four tracks originate from the same vertex near the interaction region. As a result, $p p \rightarrow Z Z \rightarrow 4 l$ is the major irreducible background that survives all the possible selection requirements [34, 35]. We emphasize that, while it is possible to understand gross features of the backgrounds, it is conceivable that subtle (but relevant) details of the angular distributions due to background processes may weakly depend on the exact background composition. Such an exact composition is hard to predict theoretically with any degree of confidence but, fortunately, this is not necessary. Indeed, this problem can be solved by using sideband analysis. This possibility is incorporated into our analysis discussed in the next section, but its detailed study is beyond the scope of the present paper. Here, we restrict ourselves to $p p \rightarrow Z Z \rightarrow 4 l$ as the only background source and simulate it using Madgraph [36].

In order to illustrate MC simulation, compare it to the derived angular distributions, provide examples of the data analysis techniques, and understand the statistical power of the proposed approaches; we choose seven scenarios which cover all spin and parity combinations. They are described in Table I] Note that for the $2^{+}$graviton-like resonance, we consider two models with different couplings to matter and gauge fields. The distributions of all five angles $-\cos \theta^{*}, \Phi_{1}, \cos \theta_{1}, \cos \theta_{2}$, and $\Phi-$ for the seven models in Table I and the $p p \rightarrow Z Z \rightarrow 4 l$ background process are shown in Fig. 2. These distributions were generated with our program assuming that the resonance mass is $m_{X}=250 \mathrm{GeV}$. Throughout the paper we consider $\sqrt{s}=14 \mathrm{TeV}$ proton-proton collisions and use the CTEQ6L1 parton distribution functions [37, 38]. Note that the distributions of $\cos \theta_{1}$ and $\cos \theta_{2}$ are identical and are combined in one plot in each case. Projections of the ideal angular distributions derived in the previous sections agree well with simulated distributions. A glance at Fig. 2 suggests that different hypothesis about resonance quantum numbers can be efficiently separated if all five angles are analyzed simultaneously. Of course, correlations in the multi-dimensional space of all angles are important for full separation power and those correlations cannot be easily illustrated. We fully exploit those correlations in the angular analysis discussed in the next section.

Our MC generation is performed stand-alone but, since unweighted events are produced, it is easy to incorporate it into a software framework that includes full detector simulation. This is achievable in the same way as for Madgraph 
TABLE I: The list of scenarios chosen for the analysis of the production and decay of an exotic $X$ particle with quantum numbers $J^{P}$. For the two $2^{+}$cases, the superscripts $m$ (minimal) and $L$ (longitudinal) distinguish two scenarios, as discussed in the last column. When relevant, the relative fraction of $g g$ and $q \bar{q}$ production is taken to be $1: 0$ at $m_{X}=250 \mathrm{GeV}$ and $3: 1$ at $m_{X}=1 \mathrm{TeV}$. The spin-zero $X$ production mechanism does not affect the angular distributions and therefore is not specified.

\begin{tabular}{cccc}
\hline \hline scenario $\left(J^{P}\right)$ & $X \rightarrow Z Z$ decay parameters & $X$ production parameters & comments \\
\hline $0^{+}$ & $a_{1} \neq 0$ in Eq. (2) & $g g \rightarrow X$ & SM Higgs-like scalar \\
$0^{-}$ & $a_{3} \neq 0$ in Eq. (2) & $g g \rightarrow X$ & pseudo-scalar \\
$1^{+}$ & $g_{12} \neq 0$ in Eq. (4) & $q \bar{q} \rightarrow X: \rho_{11}, \rho_{12} \neq 0$ in Eq. (9) & exotic pseudo-vector \\
$1^{-}$ & $g_{11} \neq 0$ in Eq. (4) & $q \bar{q} \rightarrow X: \rho_{11}, \rho_{12} \neq 0$ in Eq. (9) & exotic vector \\
$2_{m}^{+}$ & $g_{1}^{(2)}=g_{5}^{(2)} \neq 0$ in Eq. (5) & $g g \rightarrow X: g_{1}^{(2)} \neq 0$ in Eq. (5) & Graviton-like tensor with minimal couplings \\
& & $q \bar{q} \rightarrow X: \rho_{21} \neq 0$ in Eq. (10) & (10) \\
$2_{L}^{+}$ & $c_{2} \neq 0$ in Eq. (6) & $g g \rightarrow X: g_{2}^{(2)}=g_{3}^{(2)} \neq 0$ in Eq. (5) & Graviton-like tensor longitudinally polarized \\
& & $q \bar{q} \rightarrow X: \rho_{21}, \rho_{22} \neq 0$ in Eq. (10) & and with $J_{z}=0$ contribution \\
$2^{-}$ & $g_{8}^{(2)}=g_{9}^{(2)} \neq 0$ in Eq. (5) & $g g \rightarrow X: g_{1}^{(2)} \neq 0$ in Eq. (5) & "pseudo-tensor" \\
& & $q \bar{q} \rightarrow X: \rho_{21}, \rho_{22} \neq 0$ in Eq. (10) & \\
\hline \hline
\end{tabular}

interfaced through Pythia [39]. However, to illustrate effects of realistic detector response, we employ a simplified technique not attached to any particular experiment.

Note that, with our choice of the final state, we require measurements of the four-momenta of all charged leptons for complete reconstruction of the event kinematics, including boosts to the rest frames of $X$ and $Z \mathrm{~s}$, where the production and helicity angles are defined. In an experiment like ATLAS or CMS, the four-momentum of the charged lepton is reconstructed from its track in the detector and there are two main effects that influence these measurements: (1) deviation of the five measured track parameters, transverse momentum $\left(p_{T}\right)$, direction $(\phi, \theta)$, and distance to the origin $\left(d_{x y}, d_{z}\right)$, from their true values, and (2) non-uniform efficiency of particle detection across geometric and kinematic parameters. We model the detector response in the following way. First, we assume that, for typical track momenta of order $50-250 \mathrm{GeV} / c$ considered in our examples, the track parameter resolution can be identified with the typical ATLAS or CMS tracker system resolution. Throughout this paper, we model detector resolution using the recently reported CMS track resolution parameters [40], obtained from analysis of cosmic ray data. For the three track parameters $\left(p_{T}, \phi, \theta\right)$, we apply conservative Gaussian random smearing with an rms $\Delta p_{T}=0.025 \times p_{T}+0.0001 \times p_{T}^{2}$ $(\mathrm{GeV} / c), \Delta \phi=\Delta \theta=0.001$ ( $\mathrm{rad})$, and neglect resolution effects on the track origin. Within this simplified model of detector response, we observe that production and helicity angles can be measured with a typical resolution of the order of $\sim 0.01 \mathrm{rad}$. This resolution is rather good and does not infringe on our ability to perform angular analysis. Note that because production and helicity angles are defined in Lorentz frames which differ from the laboratory frame, they are affected by uncertainties in all track parameters and, most importantly, by $p_{T}$ uncertainties.

The second detector effect is the non-uniform reconstruction efficiency. We model it in a simple way assuming that only tracks produced centrally can be measured. We also assume that the detection efficiency does not change within the detector acceptance. Hence, the acceptance function is given by the step-function

$$
\mathcal{G}\left(\theta^{*}, \Phi_{1}, \theta_{1}, \theta_{2}, \Phi\right)=\prod_{i=1,2 ; q= \pm 1} \theta\left(\left|\eta_{\max }\right|-\left|\eta_{i, q}\left(\theta^{*}, \Phi_{1}, \theta_{1}, \theta_{2}, \Phi\right)\right|\right),
$$

where $\eta_{i, \pm}=\ln \cot \left(\theta_{i \pm} / 2\right)$ is the pseudorapidity of a lepton with the charge $q= \pm$ that originates from the decay of the $i$ 'th $Z$ boson, $\left|\eta_{\max }\right|=2.5$ is the maximal pseudorapidity that the tracker can reconstruct, and the product runs over the four leptons in the $X$ decay.

Given the simple form of the acceptance function, it is straightforward to understand the effect of detector acceptance on angles measured in the laboratory frame, but their effect on the production and helicity angles is far less obvious. Indeed, the rapidity of the lepton $i$ is a function of all production and helicity angles; as the result, Eq. (39) cuts out a complicated region in the five-dimensional space of production and helicity angles and makes the efficiency dependent on those angles. For example, if $X$ and the $Z$ bosons are produced at rest in the laboratory frame (this, of course, requires $m_{X}=2 m_{Z}$ ), the polar angles of the four final state leptons $\theta_{1+, 1-, 2+, 2-}$ that appear in Eq. (39) can be expressed through the five angles characterizing the decay as (with the convention $\Phi_{2} \equiv \Phi+\Phi_{1}$ )

$$
\mp \cos \theta_{i \pm}=\cos \theta^{*} \cos \theta_{i}+\sin \theta^{*} \sin \theta_{i} \cos \Phi_{i} .
$$

However, in general, $X$ is boosted in the laboratory frame and the $Z$ bosons are boosted in the $X$ frame. This introduces an additional transformation of the four $\theta_{i \pm}$ angles. We illustrate the effect of the lepton rapidity cut on 

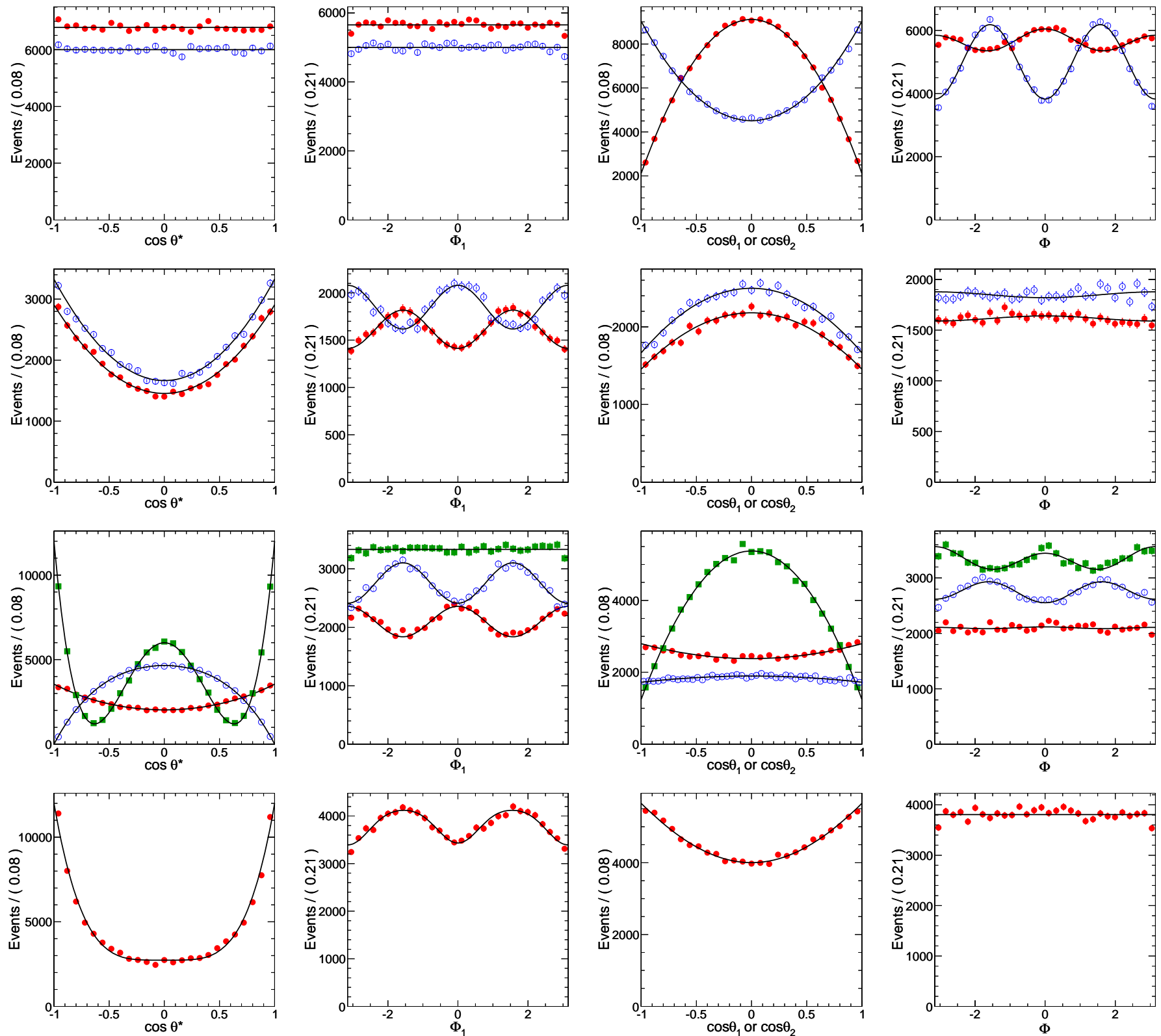

FIG. 2: Distribution of the $\cos \theta^{*}$ (left), $\Phi_{1}$ (second from the left), $\cos \theta_{1}$ and $\cos \theta_{2}$ (second from the right), and $\Phi$ (right) generated for $m_{X}=250 \mathrm{GeV}$ with the program discussed in the text (unweighted events shown as points with error bars) and projections of the ideal angular distributions given in the text (smooth lines). The four sets of plots from top to bottom show the models discussed in Table $\llbracket$ for spin-zero $0^{+}$and $0^{-}$(top), spin-one $1^{+}$and $1^{-}$(second row from top), spin-two $2_{m}^{+}, 2_{L}^{+}$, and $2^{-}$(third row from top), and the bottom row shows distributions in background generated with Madgraph (points with error bars) and empirical shape (smooth lines). The $J^{+}$distributions are shown with solid red points and $J^{-}$distributions are shown with open blue points, while the $2_{m}^{+}$and $2_{L}^{+}$are shown with red circles and green squares, respectively.

production angles in Fig. 3. where we plot the distributions of $\theta^{*}$ and $\Phi_{1}$ production angles for the spin-zero particle $X$. If these distributions are measured with the "ideal" $(4 \pi)$ detector, the results are flat. Hence, the non-trivial shapes of these distributions shown in Fig. 3 are entirely due to an acceptance effect.

It is evident from Fig. 3 that the acceptance effects are very important in the analysis of data. They have to be taken into account explicitly, otherwise the results of the analysis will be biased. This can be easily done in our MC simulation program on an event-by-event basis using the acceptance function in Eq. (39), where we reject events if at least one lepton exceeds the maximal pseudorapidity. It is also possible, but much harder, to incorporate this acceptance function into the likelihood function that is discussed in the next section. However, as we explain now, 

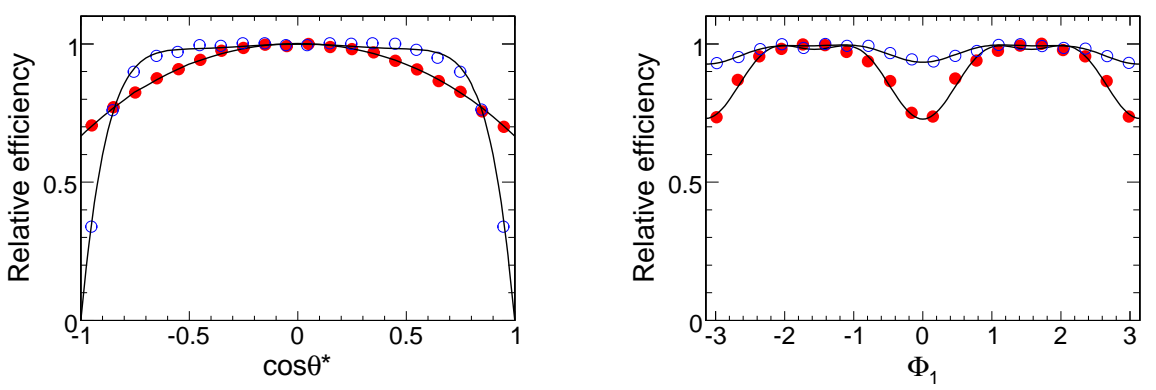

FIG. 3: Distribution of the $\cos \theta^{*}$ (left) and $\Phi_{1}$ (right) for the case of spin-zero resonance production $g g \rightarrow X \rightarrow Z Z$. The mass of the resonance is $m_{X}=250 \mathrm{GeV}$ (solid points) and $1 \mathrm{TeV}$ (open points). Detector acceptance effects are taken into account, see text for details. Lines show empirical parameterization.

certain simplifications in the treatment of the acceptance function are possible. These simplifications allow us to derive a probability distribution that accounts for major acceptance effects.

The first of the simplifying assumptions is easy to understand. Suppose $X$ is a heavy resonance. Then, $Z$ bosons from $X$ decays are strongly boosted and decay into highly collimated "leptonic jets". Therefore, as the $X$ mass increases, in the laboratory frame the decays look exceedingly pencil-like, with all the leptons aligned with directions of their parent $Z$ bosons. It is then clear that the detection efficiency of the leptons is highly correlated with the $Z$-production angle $\theta^{*}$ but not with the other angles. If the $X$ boson has an intermediate mass, the story becomes more complex and acceptance effects in other angles should be expected. However, it is reasonable to expect those effects to be moderate. We can check these features of the acceptance with the MC simulation. From Fig. 3 it is evident that with the increase in the mass of the particle $X$ the acceptance effect in $\cos \theta^{*}$ gets more pronounced while it is indeed reduced in the other angles.

The second simplifying assumption is based on the observation that distributions of the helicity angles $\left(\cos \theta_{1}, \cos \theta_{2}, \Phi\right)$ are independent of the production mechanism. Hence, there are no acceptance effects in the helicity angles provided that $\cos \theta^{*}$ and $\Phi_{1}$ are random, as in the case of spin-zero particle production. In a more general case, acceptance effects on the helicity angles are expected to be rather mild.

\section{DATA ANALYSIS}

In this section, we illustrate the application of the full angular analysis formalism by considering the production of a resonance $X$ and its subsequent decay to two $Z$ bosons. We use the MC simulation discussed in the previous section to generate both signal and background events, and apply a multivariate fitting technique discussed later in this section to extract as much information about the produced resonances as possible. We point out that, because our analysis is general and because it is not based on any particular model of BSM physics, it is not possible to accurately predict how many $X$ particles are produced, for a given luminosity. Instead, we find it more reasonable to assume a certain number of signal and background events, reconstructed by an LHC experiment. This number of events should be sufficiently large to enable meaningful angular analysis, yet it should be small enough, to be relevant for the situation soon after the resonance observation. The statistical power of the method can be easily extrapolated to a different number of resonance events.

As we explain below, we perform two different analyses in this paper, for which we choose to have 30 and 150 signal events, respectively. Roughly, this corresponds [34, 35] to the number of SM Higgs boson events $p p \rightarrow H \rightarrow Z Z \rightarrow 4 l^{ \pm}$, $l=e, \mu$, detected by one LHC experiment with 5 or $25 \mathrm{fb}^{-1}$ integrated luminosity, for a Higgs boson mass $m_{H}=250$ $\mathrm{GeV}$, at a proton-proton center-of-mass energy of $14 \mathrm{TeV}$. Of course, as we pointed out a few times already, the actual production rate of exotic particles may be larger or smaller; we choose 30 and 150 events merely for illustration purposes.

We consider two mass points, the low mass $m_{X}=250 \mathrm{GeV}$ and the relatively high mass $m_{X}=1 \mathrm{TeV}$. The background size is chosen according to the above LHC integrated luminosity convention, which leaves the high-mass scenario $m_{X}=1 \mathrm{TeV}$ with essentially no background, and the low-mass scenario with about 24 background events in the $m_{X}$ mass window between 230 and $270 \mathrm{GeV}$ [34, 35]. The exact number of background events is not crucial for our analysis because we are mostly interested in understanding how well different signal hypotheses can be disentangled, rather than how well a signal can be separated from the background.

We now imagine that 30 non-SM resonance-like events have been experimentally observed and we need to understand their origin. As the very first step, we may want to find out if the observed events are described by a resonance with 
particular quantum numbers and a particular structure of interactions with the SM particles. An efficient way to do this is to compute a confidence level at which one hypothesis about resonance quantum numbers can be separated from another hypothesis. For example, we may ask if an observed resonance looks more like a SM Higgs boson or more like a graviton with minimal couplings to SM fields. We use our MC event simulation to check how well separation of the two hypotheses can be done in practice. Once we have a good idea about the nature of the resonance $X$, we can proceed to a more statistically demanding analysis whose goal is to determine all parameters which describe the angular distributions within a particular hypothesis. Then, one may attempt to interpret those parameters within a particular model of BSM physics.

Note that the second approach clearly supersedes the first one and is the most optimal way to analyze data. Indeed, in the extreme version of the second approach, everything - mass, width, spin, parity, and polarization parameters of the particle $X$ - is obtained through a single fitting process. The shortcoming of this approach is that it is very demanding statistically, since a large sample of events is required to enable simultaneous determination of a large number of signal parameters with decent precision. For the illustration of the second approach, we assume that 150 signal events are available.

We note that our methods are general enough to implement either of the two approaches described above. The only practical difference in application of these methods is that the polarization parameters are either fixed or left as free parameters in the fit. Since it is not practical to cover the continuous spectrum of possible scenarios in this paper, we choose several examples to illustrate these approaches. These scenarios are specified in Table \.

Our studies are encouraged by the multivariate techniques developed for polarization studies in $B$-physics [41], where more than two dozen parameters describing angular and mass distributions are extracted in a simultaneous fit to a sample of several hundred events. We use an unbinned extended maximum-likelihood (ML) fit [41, 42] to extract simultaneously the signal and background yields, the background shape, and the parameters of the signal angular distributions. The likelihood function for $N$ candidate events is written as

$$
\mathcal{L}=\exp \left(-\sum_{J=1}^{3} n_{J}-n_{\mathrm{bkg}}\right) \prod_{i}^{N}\left(\sum_{J=1}^{3} n_{J} \times \mathcal{P}_{J}\left(\boldsymbol{x}_{i} ; \boldsymbol{\zeta}_{J} ; \boldsymbol{\xi}\right)+n_{\mathrm{bkg}} \times \mathcal{P}_{\mathrm{bkg}}\left(\boldsymbol{x}_{i} ; \boldsymbol{\xi}\right)\right),
$$

where $n_{J}$ is the number of signal events for each resonance spin $J, n_{\mathrm{bkg}}$ is the number of background events and $\mathcal{P}\left(\boldsymbol{x}_{i} ; \boldsymbol{\zeta} ; \boldsymbol{\xi}\right)$ is the probability density function for signal or background. It is assumed that only one resonance is observed in a given mass window and the three yields, $n_{J}$, allow one to test different hypotheses. For this reason, no interference between the different resonances is considered. Alternatively, we can consider a signal event yield $n_{\text {sig }}$ in place of the three $n_{J}$ if we assume that mass distributions of resonances with different spins are identical and a single angular distribution can be written that incorporates angular distributions for all three spins as limiting cases. An example of the latter approach is given in Eq. (38) which describes the general spin-two distribution in four angles, but also includes both Eqs. (35) and (37) written for spin-zero and spin-one.

Each event candidate $i$ is characterized by a set of six observables $\boldsymbol{x}_{i}=\left\{m_{z z}, \cos \theta^{*}, \Phi_{1}, \cos \theta_{1}, \cos \theta_{2}, \Phi\right\}_{i}$. The number of observables can be extended or reduced, depending on the desired fit. The signal polarization parameters $\left\{f_{\lambda_{1} \lambda_{2}}, \phi_{\lambda_{1} \lambda_{2}}, f_{z m}\right\}$ are collectively denoted by $\boldsymbol{\zeta}_{J}$, and the remaining parameters by $\boldsymbol{\xi}$. When several decay channels must be combined, the joint likelihood in Eq. (41) is the natural way to achieve this. In the current analysis, a combination of all lepton channels with electrons and muons in the $Z Z$ decays is assumed, but it can be easily extended to include hadronic decays of $Z$ bosons as well.

For the signal, the probability density function $\mathcal{P}_{J}\left(\boldsymbol{x}_{i} ; \boldsymbol{\zeta}_{J} ; \boldsymbol{\xi}\right)$ is taken to be a product of a function of the angles and a function of the resonance mass. For the background, the probability density function is a product of uncorrelated functions for each observable. The assumption of small correlations among the discriminating variables, except for angles where relevant, can be validated in the selected data sample by evaluating correlation coefficients and is further tested with generated experiments. As long as the results of the generated experiments are unbiased, small correlations are acceptable. Since the parameterization of the background probability density function also depends on detector effects, similarly to the signal, experiment-specific treatment will, eventually, be required. It is an approximation of our approach that the background angular distributions are not correlated, which we find acceptable for the current analysis.

The angular probability density function for the signal is the ideal distribution in Eq. (28), multiplied by an empirically determined acceptance function $\mathcal{G}\left(\cos \theta^{*}, \Phi_{1}, \cos \theta_{1}, \cos \theta_{2}, \Phi ; Y_{X}\right)$, which can be evaluated independently for each event with the $X$ particle rapidity $Y_{X}$. We approximate the acceptance function by $\mathcal{G}=\mathcal{G}^{(1)}\left(\cos \theta^{*}\right) \times \mathcal{G}^{(2)}\left(\Phi_{1}\right)$, neglecting all correlations between different angles; the justification for this approximation was given in the previous section. The parameterization of the signal acceptance functions $\mathcal{G}^{(1,2)}$ is compared with simulated distributions in Fig. 3. The acceptance function constructed along these lines can be included in the overall probability density function.

It is important to point out that one can do a better job if all that is needed is to understand how well a particular hypothesis describes experimental data. Since we test a specific hypothesis, ratios of helicity amplitudes are fixed. 

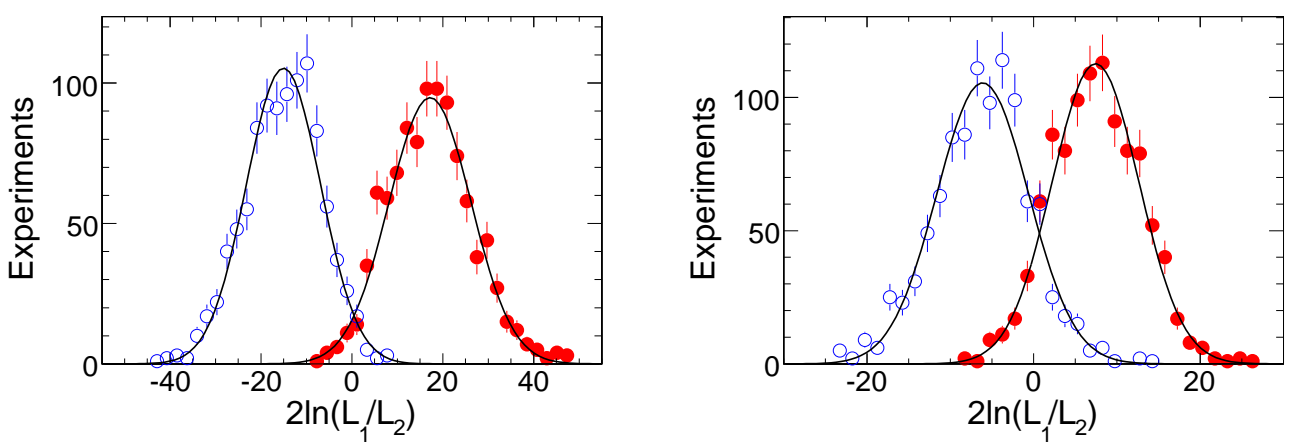

FIG. 4: Distribution of $2 \ln \left(\mathcal{L}_{1} / \mathcal{L}_{2}\right)$ with the likelihood $\mathcal{L}$ evaluated for two models $k=1,2$ and shown for 1000 generated experiments with the MC events generated according to model one ( $k=1$, open dots) and model two $(k=2$, solid dots). Left plot: $0^{+}$vs. $0^{-}$; right plot: $0^{+}$vs. $2_{m}^{+}$. Effective signal hypothesis separation power $\mathcal{S}$ is 4.1 (left plot) and 2.8 (right plot).

Hence, it is possible to determine the likelihood function numerically through the MC simulation of the full fivedimensional angular distribution, including the detector effects, though averaged over the $Y_{X}$ values. Effectively, this requires storing a normalized five-dimensional histogram with fine binning. Dependence on $Y_{X}$ could in principle be tabulated as well. A similar approach can be used for background parameterization.

However, it is more difficult to employ MC computation of the signal probability density, if we want to treat all the parameters, including helicity fractions, as free parameters in the fit. A straightforward use of the MC program would require generating and storing probability distributions for a large set of independent polarization parameters. Clearly, for a large enough number of parameters to be fitted, this procedure becomes impractical. Instead, one could factorize the angular distribution into a sum of simple terms expressed as the product of a function of the angles and a function of the polarization parameters, as we have illustrated for the spin-zero case in Eq. (35). A single independent MC simulation for the angular dependencies of each such term can be performed and the results can be combined to produce a normalized angular parameterization with free parameters. However, for the purpose of this paper, we find the simplified approach to be acceptable since, as we have verified with our MC program, biases in the results are small.

The only other kinematic observable that is used in the fit in addition to the angles is the $Z Z$ invariant mass $m_{Z Z}$. The background $m_{z Z}$ distribution is parameterized with a polynomial and its shape can be left unconstrained in the fit. We make the following assumptions about the mass and the width of the $X$ resonance. We assume that its width is negligible compared to the detector resolution effects, which are estimated to be about $3.5 \mathrm{GeV}$ at $m_{X}=250 \mathrm{GeV}$ and $23 \mathrm{GeV}$ at $m_{X}=1 \mathrm{TeV}$ with our simulation. The exact value of the resolution is only relevant for signal and background separation at the lower mass; the values quoted above are typical for both muon and electron final states. Hence, we describe the signal mass distribution with a Gaussian function of a known width. Should the resonance have a finite width that is larger than detector resolution, we can incorporate it into our analysis by replacing the Gaussian function with a convolution of the Breit-Wigner and Gaussian distributions. However, since the mass distribution has little, if any, influence on the resonance spin and its couplings to SM fields, a detailed study of mass resolution issues is outside the scope of this paper.

The mass of the resonance $m_{X}$ is assumed to be known and is fixed in the fit. This assumption only affects the signal and background separation and requires certain care in the interpretation of the discovery significance. Detailed study of this interpretation is also outside the scope of this paper, but we note that, as Fig 2 suggests, angular analysis improves the signal-background separation. As an example, consider 30 events due to the SM Higgs-like resonance at $250 \mathrm{GeV}$. If only $m_{z z}$ is employed in the fit, the measured signal significance is $5.7 \sigma$ and it increases by nearly twenty percent if also angular variables are included in the fit. Here significance is calculated from $2 \ln \left(\mathcal{L}_{1} / \mathcal{L}_{2}\right)$ using the likelihood ratio for two scenarios with signal and with pure background, analogous to the technique discussed below, and corresponds to the Gaussian probability for pure background to fluctuate to the observed signal yield. The only constraint on background size comes from data sidebands included in the fit.

We are now in position to discuss the results of our analysis. As an illustration of the first approach, in Fig. 4, we plot the quantity $2 \ln \left(\mathcal{L}_{1} / \mathcal{L}_{2}\right)$ for two sets of $\mathrm{MC}$ experiments generated with the models $0^{+}$and $0^{-}$(left plot) or $0^{+}$and $2_{m}^{+}$(right plot), as listed in Table [1. Each experiment has on average 24 background and 30 signal events, Poisson distributed. These events are generated at $m_{X}=250 \mathrm{GeV}$ in 1000 statistically independent experiments. The likelihood $\mathcal{L}_{k}$ in Eq. (41) is evaluated independently for each hypothesis $k$. The discussion of the statistical interpretation of $2 \ln \left(\mathcal{L}_{1} / \mathcal{L}_{2}\right)$ was given recently in Ref. [17]. The distribution of $2 \ln \left(\mathcal{L}_{1} / \mathcal{L}_{2}\right)$ is expected to peak at positive values for events generated according to model one (on average $\mathcal{L}_{1}>\mathcal{L}_{2}$ ) and at negative values for events 
TABLE II: Results of the hypothesis separation of the seven scenarios listed in Table 1 for $m_{X}=250$ GeV. The five numbers quoted in each case correspond to the $1 \mathrm{D} / 2 \mathrm{D} / 3 \mathrm{D} / 4 \mathrm{D} / 5 \mathrm{D}$ angular information used in the fit, as discussed in the text. The results are reported as an equivalent separation of two single-width Gaussian distributions in terms of the number of Gaussian standard deviations between the two peaks.

\begin{tabular}{|c|c|c|c|c|c|c|}
\hline & $0^{-}$ & $1^{+}$ & $1^{-}$ & $2_{m}^{+}$ & $2_{L}^{+}$ & $2^{-}$ \\
\hline$\overline{0^{+}}$ & $0.0 / 0.0 / 3.9 / 4.1 / 4.1$ & $0.8 / 1.0 / 1.8 / 1.9 / 2.3$ & $0.9 / 1.0 / 2.5 / 2.6 / 2.6$ & $0.8 / 0.9 / 2.4 / 2.5 / 2.8$ & $2.6 / 2.6 / 0.0 / 2.6 / 2.6$ & $1.6 / 1.7 / 2.4 / 3.0 / 3.3$ \\
\hline $0^{-}$ & & $0.8 / 1.2 / 2.8 / 3.0 / 3.1$ & $0.9 / 1.0 / 2.5 / 2.8 / 3.0$ & $0.8 / 0.8 / 1.7 / 2.0 / 2.4$ & $2.9 / 2.9 / 4.1 / 4.8 / 4.8$ & $1.6 / 1.7 / 2.0 / 2.7 / 2.9$ \\
\hline $1^{+}$ & & & $0.0 / 1.1 / 1.1 / 1.2 / 2.2$ & $0.1 / 1.2 / 1.3 / 1.4 / 2.6$ & $2.8 / 2.8 / 1.9 / 3.5 / 3.6$ & $2.5 / 2.4 / 1.2 / 2.7 / 2.9$ \\
\hline $1^{-}$ & & & & $0.1 / 0.1 / 1.3 / 1.5 / 1.8$ & $2.8 / 2.9 / 2.5 / 3.8 / 3.8$ & $2.5 / 2.6 / 0.6 / 2.8 / 3.4$ \\
\hline $2_{m}^{+}$ & & & & & $2.9 / 2.9 / 2.6 / 3.6 / 3.8$ & $2.3 / 2.5 / 0.5 / 2.5 / 3.2$ \\
\hline $2_{L}^{+}$ & & & & & & $3.6 / 3.6 / 2.5 / 4.2 / 4.3$ \\
\hline
\end{tabular}

TABLE III: Results of the hypothesis separation of the seven scenarios for $m_{X}=1 \mathrm{TeV}$ with the same notation as in Table II

\begin{tabular}{|c|c|c|c|c|c|c|}
\hline & $0^{-}$ & $1^{+}$ & $1^{-}$ & $2_{m}^{+}$ & $2_{L}^{+}$ & $2^{-}$ \\
\hline $0^{+}$ & $0.0 / 0.0 / 3.9 / 4.0 / 4.1$ & $0.4 / 0.6 / 2.2 / 2.2 / 2.4$ & $0.3 / 0.5 / 2.2 / 2.1 / 2.4$ & $1.2 / 1.1 / 2.8 / 2.7 / 2.8$ & $1.0 / 1.0 / 0.0 / 1.2 / 1.2$ & $1.2 / 1.0 / 2.0 / 2.7 / 2.8$ \\
\hline $0^{-}$ & & $0.5 / 0.9 / 2.8 / 2.9 / 3.1$ & $0.5 / 0.8 / 2.7 / 2.9 / 3.1$ & $1.4 / 1.5 / 1.4 / 2.1 / 2.1$ & $1.1 / 1.1 / 4.0 / 4.3 / 4.5$ & $0.8 / 0.9 / 2.9 / 2.8 / 2.9$ \\
\hline $1^{+}$ & & & $0.0 / 1.1 / 1.2 / 1.2 / 2.3$ & $0.9 / 0.9 / 2.0 / 2.0 / 2.3$ & $1.3 / 1.4 / 2.1 / 2.4 / 2.6$ & $1.3 / 1.3 / 1.1 / 1.8 / 2.0$ \\
\hline $1^{-}$ & & & & $0.9 / 0.9 / 2.0 / 2.0 / 2.1$ & $1.3 / 1.3 / 2.2 / 2.5 / 2.7$ & $1.4 / 1.6 / 0.0 / 1.4 / 2.2$ \\
\hline $2_{m}^{+}$ & & & & & $1.7 / 1.7 / 3.0 / 3.2 / 3.2$ & $2.1 / 2.0 / 2.1 / 2.6 / 2.6$ \\
\hline $2_{L}^{+}$ & & & & & & $1.5 / 1.5 / 2.1 / 3.3 / 3.3$ \\
\hline
\end{tabular}

generated according to model two (on average $\mathcal{L}_{2}>\mathcal{L}_{1}$ ). From Fig. 4 , we extract the quantity $\mathcal{S}$ in the following way. We find the point beyond which the right-side tail of the left histogram and the left-side tail of the right histogram have equal areas. These areas correspond to the one-sided Gaussian probability outside of the $\mathcal{S} / 2 \sigma$ range. If the two histograms in Fig. 4 were perfectly Gaussian distributed with unit width, then $\mathcal{S}$ corresponds to the separation between the peaks of the two distributions.

The above procedure is repeated for all combinations of seven hypotheses listed in Table I, for $m_{X}=250 \mathrm{GeV}$ and $m_{X}=1 \mathrm{TeV}$, and results are presented in Tables [I] and III. In those tables, we show the increase in the separation power between two hypotheses if more information about angular distributions is included in the likelihood fit. It is natural to refer to a likelihood fit that includes $n$ angular variables as $n$-dimensional or $n \mathrm{D}$; the results of the 1D, 2D, 3D, 4D, and 5D fits are shown in Tables II and III. In the 1D fit, only the $\cos \theta^{*}$ angular distribution is used; in the $2 \mathrm{D}$ fit, the $\left(\cos \theta^{*}, \Phi_{1}\right)$ distribution is included; in the $3 \mathrm{D}$ fit, we have $\left(\cos \theta_{1}, \cos \theta_{2}, \Phi\right)$; in the $4 \mathrm{D}$ fit, the $\left(\cos \theta^{*}, \cos \theta_{1}, \cos \theta_{2}, \Phi\right)$ distribution is used; and finally in $5 \mathrm{D}$ fit, the $\left(\cos \theta^{*}, \Phi_{1}, \cos \theta_{1}, \cos \theta_{2}, \Phi\right)$ distribution is employed. Statistical significance achieved in the 4D fit is already close to the statistical significance of the optimal analysis which employs all five angles. We note that because we generate a finite number of experiments (1000), the statistical uncertainties on the values of $\mathcal{S}$ reported in Tables II and III are typically in the range $0.1-0.2$. This explains some of the small discrepancies in the values quoted. For example, one could expect the separation of $0^{+}$ and $0^{-}$hypotheses to be the same in $3 \mathrm{D}, 4 \mathrm{D}$, and $5 \mathrm{D}$ cases. However, statistical fluctuations and better background suppression make the 4D and 5D cases look better.

It is clear from Tables $\Pi$ and $\amalg$ that the multidimensional angular analysis allows efficient separation of different spin hypotheses and, therefore, is a very powerful approach to spin determination. While separations $\mathcal{S}=2$ are typically achieved, there are important cases when larger separations are possible. In particular, we observe $\mathcal{S}=4$ for the separation of $0^{+}$and $0^{-}$hypotheses, which might be useful even for the SM Higgs boson if its mass exceeds $2 m_{z}$. Also, the separation of the spin-two with the minimal couplings hypothesis from the spin-two with longitudinal amplitude dominance hypothesis is close to $\mathcal{S}=3$, for any production mechanism scenario. In all cases, the results of the $5 \mathrm{D}$ fit provide the best separation power and supersede the other fits. Inclusion of the $\Phi_{1}$ angle in the $2 \mathrm{D}$ or $5 \mathrm{D}$ fit, compared to the $1 \mathrm{D}$ or $4 \mathrm{D}$ fit, usually adds little information, except for the separation of the two spin-one hypotheses which have identical $\cos \theta^{*}$ distributions. Therefore, the separation power obtained from the angular analysis with all five angles is very close to the separation power of the $4 \mathrm{D}$ fit.

A glance at Tables 【I and III is sufficient to recognize that there are cases when different hypotheses about the nature of the resonance cannot be separated $(\mathcal{S}=0)$. For example, the production angles $\cos \theta^{*}$ and $\Phi_{1}$ provide no separation between the two spin-zero scenarios $\left(0^{+}\right.$and $\left.0^{-}\right)$due to the absence of spin correlations. At the same time, the three helicity angle $\left(\cos \theta_{1}, \cos \theta_{2}, \Phi\right)$ distributions are identical for the $0^{+}$and $2_{L}^{+}$hypotheses, therefore the $3 \mathrm{D}$ case results in $\mathcal{S}=0$ as well. The only source of separation in this case is the production angle. We have not 

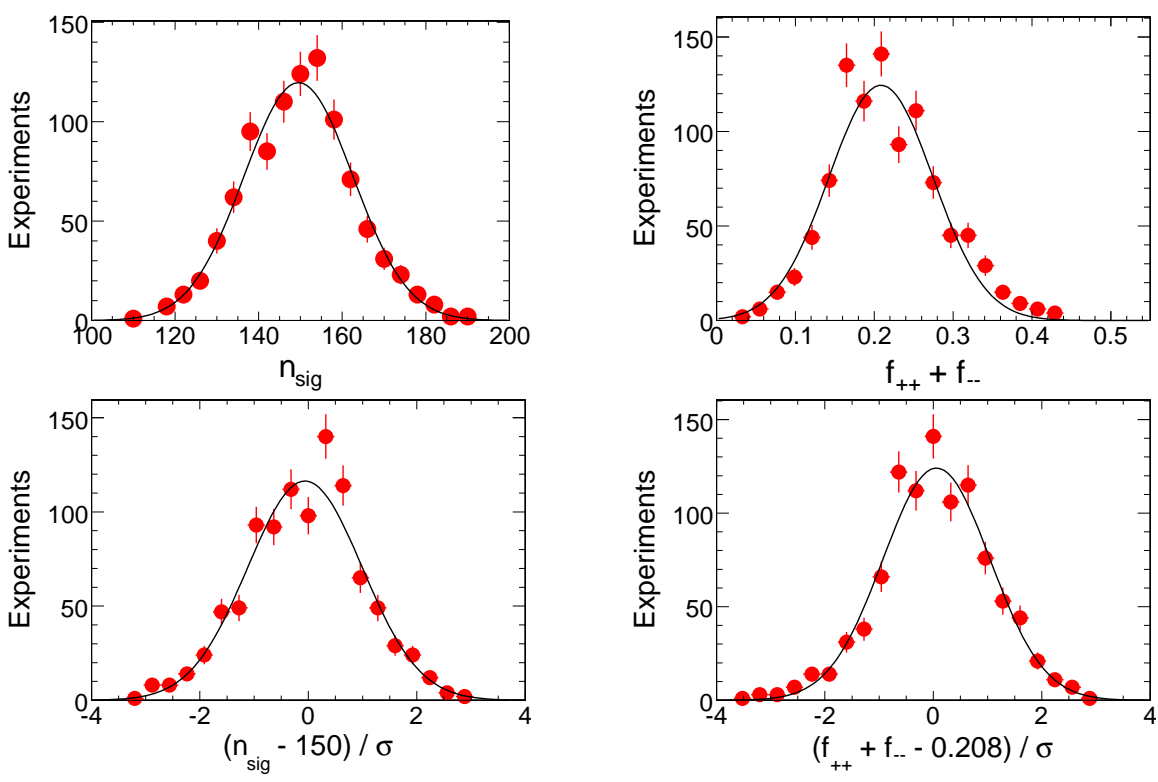

FIG. 5: Top: distribution of the number of fitted signal events $n_{\text {sig }}$ (left) and the fraction of transverse component in the decay amplitude $\left(f_{++}+f_{--}\right)$(right) in 1000 generated experiments with $0^{+}$hypothesis corresponding to Table IV Bottom: distribution of the above parameters normalized by the fit errors.

considered any example with no separation from either production or helicity angles, but such situation is possible when spin-zero and spin-two scenarios with $f_{z 1}=f_{z 2}=0.4$ are considered, as we discussed earlier. A hint at this situation is observed in the decreased $\mathcal{S}$-value that characterizes the separation of $0^{+}$and $2_{L}^{+}$hypotheses when the mass of the resonance increases from $250 \mathrm{GeV}$ to $1 \mathrm{TeV}$. This is the consequence of the fact that at $1 \mathrm{TeV}$ we allow $q \bar{q}$ production. This leads to $f_{z 1}=0.25$ and $f_{z 2}=0.30$, which is closer to the unpolarized case. As the result, the $\mathcal{S}$-value decreases dramatically.

Obviously, in the above studies we could not cover all possible scenarios. Just as an example, one could consider a scenario of a non-SM Higgs-like scalar with quantum numbers $0^{+}$with $a_{1}=0, a_{2} \neq 0$ in Eq. (2). The separation of this hypothesis from the SM Higgs-like scalar will depend strongly on the mass of the resonance. As it is evident from Eq. (16), the single $A_{00}$ amplitude dominates for $m_{X}^{2} / m_{V}^{2} \gg 1$ in both cases. Therefore, separation of the two hypotheses is impossible at high mass. However, at lower mass, there is a sizable contribution of both $A_{++}$and $A_{--}$ in the SM Higgs-like case, but not in the other case. Therefore, the most optimal analysis strategy is to fit for all the polarization parameters, and then interpret the result within a particular model of BSM physics. We describe the feasibility of such fit in the remainder of this section.

The most general analysis of an observed resonance requires that the likelihood in Eq. (41) is maximized with respect to the signal yield and all signal angular parameters, $\boldsymbol{\zeta}_{J}$, as well as with respect to unconstrained background parameters $\boldsymbol{\xi}$. This requires a large number of free parameters even for signal alone. The fit delivers the most probable values of these parameters and the covariance matrix which describes their uncertainties and correlations ${ }^{3}$. In principle, it is possible to pursue this strategy within our approach, but for the fit to converge with high success rate, one may need large enough event sample. Therefore, for the illustration of the fit technique, we consider a limited procedure where only one spin hypothesis is considered at a time, but all other parameters relevant for that particular spin hypothesis are allowed to float in the fit.

In Tables $[\mathrm{IV}$ and $\mathrm{V}$, this technique is illustrated with the spin-zero hypothesis with samples generated according to the $0^{+}$and $0^{-}$scenarios, respectively. We show the results of the fit with both "perfect" and realistic detectors. In Fig. 5 we show distributions for two parameters in this analysis for a set of 1000 generated experiments. The means $\mu$ and widths $w$ of these distributions are shown in Table IV as $\mu \pm w$. These values are the most probable central values and errors of a measurement, but they are subject to statistical fluctuation in each given experiment. Normalized distributions in Fig. [5 illustrate proper error estimates in the fit. In Tables VI and VII, the result of a similar study with the spin-one hypotheses $1^{+}$and $1^{-}$are presented. Finally, in Tables [VII, VIII, and IX] we illustrate the fit

\footnotetext{
${ }^{3}$ Knowledge of the full dependence of $-2 \ln \mathcal{L}$ on all the parameters is even more beneficial.
} 
TABLE IV: Results of the fit for the free parameters of the spin-zero hypothesis with generated samples of SM Higgs-like $X$ corresponding to $0^{+}$in Table II Experiments have been generated with two $X$ masses according to two models in each case, with and without detector effects.

\begin{tabular}{|c|c|c|c|c|c|c|}
\hline & generated & $\begin{array}{r}m_{X}=250 \mathrm{Ge} \\
\text { fitte } \\
\text { without detector }\end{array}$ & d with detector & generated & $\begin{array}{r}m_{X}=1 \mathrm{TeV} \\
\text { fitte } \\
\text { without detector }\end{array}$ & with detector \\
\hline$n_{\text {sig }}$ & 150 & $150 \pm 13$ & $153 \pm 15$ & 150 & $150 \pm 12$ & $152 \pm 12$ \\
\hline$\left(f_{++}+f_{--}\right)$ & 0.208 & $0.21 \pm 0.07$ & $0.23 \pm 0.08$ & 0.000 & $0.00 \pm 0.03$ & $0.00 \pm 0.03$ \\
\hline$\left(f_{++}-f_{--}\right)$ & 0.000 & $0.01 \pm 0.13$ & $0.01 \pm 0.14$ & 0.000 & $0.00 \pm 0.02$ & $0.00 \pm 0.02$ \\
\hline$\left(\phi_{++}+\phi_{--}\right)$ & $2 \pi$ & $6.30 \pm 1.46$ & $6.39 \pm 1.54$ & $2 \pi$ & free & free \\
\hline$\left(\phi_{++}-\phi_{--}\right)$ & 0 & $0.00 \pm 1.06$ & $0.01 \pm 1.09$ & 0 & free & free \\
\hline
\end{tabular}

TABLE V: Results of the fit for the free parameters of the spin-zero hypothesis with generated samples of a pseudo-scalar $X$ corresponding to $0^{-}$as discussed in Table IV

\begin{tabular}{|c|c|c|c|c|c|c|}
\hline & generated & $\begin{array}{r}m_{X}=250 \mathrm{Ge} \\
\text { fitte } \\
\text { without detector }\end{array}$ & $\begin{array}{l}\mathrm{d} \\
\mathrm{d} \text { with detector }\end{array}$ & generated & $\begin{array}{r}m_{X}=1 \mathrm{TeV} \\
\text { fitte } \\
\text { without detector }\end{array}$ & d with detector \\
\hline$n_{\text {sig }}$ & 150 & $150 \pm 13$ & $151 \pm 15$ & 150 & $151 \pm 12$ & $150 \pm 13$ \\
\hline$\left(f_{++}+f_{--}\right)$ & 1.000 & $1.00 \pm 0.05$ & $1.00 \pm 0.06$ & 1.000 & $1.00 \pm 0.05$ & $1.00 \pm 0.06$ \\
\hline$\left(f_{++}-f_{--}\right)$ & 0.000 & $0.00 \pm 0.35$ & $0.00 \pm 0.40$ & 0.000 & $0.00 \pm 0.31$ & $-0.01 \pm 0.32$ \\
\hline$\left(\phi_{++}+\phi_{--}\right)$ & $\mathrm{N} / \mathrm{A}$ & free & free & $\mathrm{N} / \mathrm{A}$ & free & free \\
\hline$\left(\phi_{++}-\phi_{--}\right)$ & $\pi$ & $3.15 \pm 0.31$ & $3.14 \pm 0.41$ & $\pi$ & $3.15 \pm 0.31$ & $3.14 \pm 0.33$ \\
\hline
\end{tabular}

TABLE VI: Results of the fit for the free parameters of the spin-one hypothesis with generated samples of an exotic pseudovector $X$ corresponding to $1^{+}$as discussed in Table IV

\begin{tabular}{|c|c|c|c|c|c|c|}
\hline & generated & $\begin{array}{r}m_{X}=250 \mathrm{Ge} \\
\text { fitt } \\
\text { without detecto }\end{array}$ & d with detector & generated & $\begin{array}{r}m_{X}=1 \mathrm{TeV} \\
\text { fitte } \\
\text { without detector }\end{array}$ & with detector \\
\hline$n_{\text {sig }}$ & 150 & $150 \pm 13$ & $152 \pm 15$ & 150 & $151 \pm 12$ & $152 \pm 13$ \\
\hline$f_{+0}$ & 0.250 & $0.26 \pm 0.15$ & $0.26 \pm 0.17$ & 0.250 & $0.25 \pm 0.16$ & $0.25 \pm 0.15$ \\
\hline$\left(\phi_{+0}-\phi_{0-}\right)$ & 0 & $0.02 \pm 0.73$ & $0.01 \pm 0.86$ & 0 & $-0.03 \pm 0.67$ & $-0.03 \pm 0.77$ \\
\hline
\end{tabular}

TABLE VII: Results of the fit for the free parameters of the spin-one hypothesis with generated samples of an exotic vector $X$ corresponding to $1^{-}$as discussed in Table IV

\begin{tabular}{|c|c|c|c|c|c|c|}
\hline & generated & $\begin{array}{r}m_{X}=250 \mathrm{Ge} \\
\text { fitt } \\
\text { without detector }\end{array}$ & $\begin{array}{l}\text { d with detector } \\
\text { with }\end{array}$ & generated & $\begin{array}{r}m_{X}=1 \mathrm{TeV} \\
\text { fitte } \\
\text { without detector }\end{array}$ & d with detector \\
\hline$n_{\text {sig }}$ & 150 & $150 \pm 13$ & $152 \pm 16$ & 150 & $151 \pm 12$ & $152 \pm 13$ \\
\hline$f_{+0}$ & 0.250 & $0.24 \pm 0.15$ & $0.24 \pm 0.16$ & 0.250 & $0.24 \pm 0.17$ & $0.25 \pm 0.15$ \\
\hline$\left(\phi_{+0}-\phi_{0-}\right)$ & $\pi$ & $3.14 \pm 0.72$ & $3.18 \pm 0.76$ & $\pi$ & $3.14 \pm 0.71$ & $3.14 \pm 0.69$ \\
\hline
\end{tabular}

with the spin-two hypotheses for $2_{m}^{+}, 2_{L}^{+}$, and $2^{-}$, respectively, where we also restrict the hypothesis to the definite parity to reduce the number of free parameters. We used 1000 generated samples of 150 signal events each, Poisson distributed, and the corresponding number of background events, per sample. In all cases the set of observables includes $m_{Z Z}, \cos \theta^{*}, \cos \theta_{1}, \cos \theta_{2}$, and $\Phi$. There are several cases where contribution of a certain helicity amplitude is either exactly zero (e.g. $A_{00}$ in the $0^{-}$case) or negligible (e.g. $A_{++}$in the $2_{m}^{+}$case). Since measuring the phase of that amplitude becomes impossible, we leave it as "free" in the tables.

Coming back to the question of separating SM-like and non-SM-like $0^{+}$hypotheses, it is clear from Fig. 5 and Table IV that the hypothesis $a_{1}=0$ and $a_{2} \neq 0$ in Eq. (2), corresponding to $f_{++}+f_{--}=0$, can be separated with high confidence at $250 \mathrm{GeV}$, but no separation is possible at $1 \mathrm{TeV}$ due to the absence of a sizable transverse amplitude in both scenarios. As another example, the separation of the $1^{+}$and $1^{-}$scenarios in Tables II and III is related to the measurement of $\left(\phi_{+0}-\phi_{0-}\right)$ in Tables VI and VII, although there is an additional free parameter in the latter case. The signature of the minimal coupling model of a spin-two resonance decay is the dominance of the $A_{+-}$ 
TABLE VIII: Results of the fit for the free parameters of the spin-two hypothesis with positive parity with generated samples of an exotic $X$ corresponding to $2_{m}^{+}$as discussed in Table IV

\begin{tabular}{|c|c|c|c|c|c|c|}
\hline & generated & $\begin{array}{r}\quad m_{X}=250 \mathrm{GeV} \\
\text { fitte } \\
\text { without detector }\end{array}$ & d with detector & generated & $\begin{array}{r}\text {. } m_{X}=1 \mathrm{TeV} \\
\text { fitt } \\
\text { without detecto }\end{array}$ & d with detector \\
\hline$n_{\text {sig }}$ & 150 & $150 \pm 13$ & $151 \pm 16$ & 150 & $151 \pm 12$ & $153 \pm 13$ \\
\hline$f_{z 2}$ & 1.000 & $1.00 \pm 0.17$ & $0.84 \pm 0.17$ & 0.750 & $0.75 \pm 0.12$ & $0.80 \pm 0.10$ \\
\hline$f_{z 1}$ & 0.000 & $0.00 \pm 0.19$ & $0.00 \pm 0.25$ & 0.250 & $0.25 \pm 0.14$ & $0.16 \pm 0.15$ \\
\hline$f_{++}$ & 0.013 & $0.01 \pm 0.04$ & $0.00 \pm 0.05$ & 0.000 & $0.00 \pm 0.05$ & $0.00 \pm 0.05$ \\
\hline$f_{+-}$ & 0.282 & $0.28 \pm 0.04$ & $0.31 \pm 0.05$ & 0.445 & $0.44 \pm 0.06$ & $0.44 \pm 0.04$ \\
\hline$f_{+0}$ & 0.075 & $0.07 \pm 0.04$ & $0.06 \pm 0.05$ & 0.000 & $0.01 \pm 0.06$ & $0.01 \pm 0.06$ \\
\hline$\phi_{++}$ & 0 & $0.00 \pm 1.75$ & $0.04 \pm 1.76$ & 0 & free & free \\
\hline
\end{tabular}

TABLE IX: Results of the fit for the free parameters of the spin-two hypothesis with positive parity with generated samples of an exotic $X$ corresponding to $2_{L}^{+}$as discussed in Table IV

\begin{tabular}{|c|c|c|c|c|c|c|}
\hline & generated & $\begin{array}{r}m_{X}=250 \mathrm{GeV} \\
\text { fitte } \\
\text { without detector }\end{array}$ & with detector & generated & $\begin{array}{r}\text { r } m_{X}=1 \mathrm{TeV} \\
\text { fitt } \\
\text { without detecto }\end{array}$ & d with detector \\
\hline$n_{\text {sig }}$ & 150 & $150 \pm 13$ & $154 \pm 15$ & 150 & $151 \pm 12$ & $152 \pm 13$ \\
\hline$f_{z 2}$ & 0.400 & $0.40 \pm 0.07$ & $0.33 \pm 0.10$ & 0.300 & $0.30 \pm 0.08$ & $0.34 \pm 0.09$ \\
\hline$f_{z 1}$ & 0.000 & $0.00 \pm 0.03$ & $0.00 \pm 0.04$ & 0.250 & $0.25 \pm 0.07$ & $0.23 \pm 0.07$ \\
\hline$f_{++}$ & 0.104 & $0.10 \pm 0.04$ & $0.06 \pm 0.05$ & 0.000 & $0.00 \pm 0.01$ & $0.00 \pm 0.02$ \\
\hline$f_{+-}$ & 0.000 & $0.00 \pm 0.04$ & $0.04 \pm 0.05$ & 0.000 & $0.00 \pm 0.01$ & $0.00 \pm 0.03$ \\
\hline$f_{+0}$ & 0.000 & $0.00 \pm 0.02$ & $0.00 \pm 0.02$ & 0.000 & $0.00 \pm 0.02$ & $0.00 \pm 0.02$ \\
\hline$\phi_{++}$ & $\pi$ & $3.20 \pm 0.75$ & $3.17 \pm 0.71$ & $\pi$ & free & free \\
\hline
\end{tabular}

TABLE X: Results of the fit for the free parameters of the spin-two hypothesis with negative parity with generated samples of an exotic $X$ corresponding to $2^{-}$as discussed in Table IV

\begin{tabular}{|c|c|c|c|c|c|c|}
\hline & generated & $\begin{array}{r}m_{X}=250 \mathrm{Ge} \\
\text { fitte } \\
\text { without detector }\end{array}$ & with detector & generated & $\begin{array}{r}m_{X}=1 \mathrm{TeV} \\
\text { fitt } \\
\text { without detector }\end{array}$ & with detector \\
\hline$n_{\mathrm{sig}}$ & 150 & $150 \pm 13$ & $152 \pm 15$ & 150 & $151 \pm 12$ & $152 \pm 13$ \\
\hline$f_{z 2}$ & 1.000 & $1.00 \pm 0.06$ & $0.97 \pm 0.12$ & 0.750 & $0.75 \pm 0.11$ & $0.81 \pm 0.15$ \\
\hline$f_{z 1}$ & 0.000 & $0.00 \pm 0.11$ & $0.05 \pm 0.20$ & 0.250 & $0.25 \pm 0.13$ & $0.19 \pm 0.24$ \\
\hline$f_{++}$ & 0.125 & $0.12 \pm 0.06$ & $0.13 \pm 0.07$ & 0.000 & $0.00 \pm 0.07$ & $0.00 \pm 0.07$ \\
\hline
\end{tabular}

and $A_{-+}$amplitudes, while the model discussed in Refs. 21 23] predicts longitudinal $A_{00}$ amplitude dominance. This was our motivation for comparing the $2_{m}^{+}$and $2_{L}^{+}$cases. We observe that the angular distributions of the two models could be well separated based on the measurement of $f_{+-}$, as it is also evident from Fig. 2 and Tables 【, 【II. There are several cases where probability distributions of some parameter are not Gaussian; typically, this occurs when the central value of a parameter is at, or very close to, its physical boundary. In those cases, we still approximate the expectation value for this parameter and the error by $\mu \pm w$, where we extrapolate the value of $w$ from the rms, but we note that statistical meaning of the error estimated in such a way can be very different. For this reason, the proper interpretation of the values quoted in the tables in terms of the separation $\mathcal{S}$ value requires a more careful treatment which accounts for the non-Gaussian shape of all distributions.

The general conclusion from Tables IV $\mathrm{X}$ is that our analysis yields unbiased results for all the parameters that describe the angular distributions in the production and decay of a resonance $X$. Indeed, it follows from those tables that, when detector effects are neglected, the central values of all the parameters correspond to their input values. This verifies both the fit implementation and angular parameterizations.

Once detector effects are introduced, certain biases appear but they are minor. These biases come from the fact that we ignore angular correlations in both the detector acceptance function and in our parametrization of angular background distributions. Both of these parameterizations are detector-specific and would need to be modeled in each experiment. For illustration purposes, we introduced a simplified model of the acceptance function in the present 
paper. We pointed out that the sophistication of the required model for the acceptance function and the background parametrization depends on the actual mass of the observed resonance. Indeed, it is evident from Tables [IV] $X]$ that the bias is smaller at higher masses due to reduced angular correlations, as we explained earlier. The expected statistical errors of the measurements are not strongly affected by detector effects; this allows us to predict statistical precision in future measurements.

The above studies illustrate the precision on individual parameters that can be achieved with a sample of 150 signal events. These results can be easily extrapolated to an event sample of a different size. On the other hand, if an accurate precision estimate is required in a particular scenario, dedicated analysis is necessary. In the present paper, we do not include the evaluation of systematic uncertainties beyond simplified studies of resolution and acceptance detector effects, but these and other systematic effects are typically much smaller than statistical uncertainties if the signal significance is close to the discovery threshold.

\section{CONCLUSIONS}

The importance of the LHC physics program for the future of high-energy physics requires designing techniques and analysis tools that are not biased towards a particular model of beyond the Standard Model physics. While it may be impractical or impossible to do so in many complicated cases, the case of a single resonance production at the LHC is sufficiently robust to aim at the most general description, yet simple enough to make such a description possible. Motivated by this, we studied production of a single resonance at the LHC and its decay into four-lepton final states in the process $p p \rightarrow X \rightarrow Z Z \rightarrow l_{1} \bar{l}_{1} l_{2} \bar{l}_{2}$, allowing for the most general couplings of the resonance to Standard Model matter and gauge fields. Our goal was to understand if full reconstruction of the final state and the analysis of the most general angular distributions can be used to determine the spin and parity of the resonance and to constrain its couplings to vector gauge bosons, quarks, and gluons.

It is interesting to point out that, in some cases, generality of the couplings leads to new effects in the angular distributions. For example, imagine that the spin-two resonance is identified with the massive graviton whose coupling to gauge and matter fields is fixed through the energy-momentum tensor. Production of such a resonance in gluon fusion occurs only with spin projections on the collision axis $J_{z}= \pm 2$. On the other hand, if a general coupling of the spin-two resonance to gluons is considered, production with $J_{z}=0$ becomes possible as well. There are interesting consequences of this observation related to a possibility to discriminate between minimal and non-minimal couplings of the spin-two resonance through the analysis of angular distributions.

Motivated by this example, we derived the most general angular distributions of four fermions in the process $p p \rightarrow X \rightarrow V V \rightarrow f_{1} f_{1}^{\prime} f_{2} f_{2}^{\prime}$, considering spin-zero, spin-one, and spin-two resonances ${ }^{4}$. We used those angular distributions to construct the likelihood functions for the angular analysis. We wrote a Monte Carlo simulation program that describes the production of the resonance $X$ in the process $p p \rightarrow X \rightarrow Z Z \rightarrow l_{1} \bar{l}_{1} l_{2} \bar{l}_{2}$, includes all spin correlations throughout the decay chains, and employs the most general couplings of the resonance $X$ to matter and gauge fields of the Standard Model. We supplemented this analysis with a simplified (but fairly realistic) model of the detector effects. When all the pieces of our study are put together, we obtain a powerful analysis tool that allows for a realistic estimate of how much information the two LHC experiments will be able to extract from the study of angular distributions once the resonance in the process $p p \rightarrow X \rightarrow Z Z \rightarrow l_{1} \bar{l}_{1} l_{2} \bar{l}_{2}$ is observed.

We find that angular distributions provide good separation of various hypotheses about the spin of the resonance. As we illustrate in Tables II and III. with 30 fully reconstructed events a typical separation of various spin hypotheses is $\mathcal{S}=2-3 \sigma$, as defined in text, but in some cases separation as good as $4 \sigma$ is achieved. With a somewhat larger event sample, it becomes possible to determine helicity amplitude fractions that characterize resonance production and decay as well as the resonance spin from a multidimensional likelihood fit with decent precision. Model-independent determination of the helicity amplitude fractions and phases is the ultimate goal of such an analysis which can then be interpreted within any model of beyond the Standard Model physics. We provide relationships between the amplitude measurements and the fundamental coupling constants of the resonance to matter and gauge fields. Our studies show that such model-independent analysis is viable at the LHC. We look forward to its application to real LHC data.

Finally, we note that the analysis presented in this paper can be extended in a number of ways. A natural possibility is to allow hadronic final states (e.g. $Z \rightarrow q \bar{q}$ ) and/or missing energy. An obvious candidate for the latter is the decay $X \rightarrow W^{+} W^{-}$. We note that most of the discussion of the $X \rightarrow Z Z$ decays given in this paper also applies to decays $X \rightarrow W^{+} W^{-}$. However, there are important differences related to the fact that $W^{+}$and $W^{-}$are not identical particles. Consequently, Eq. (14) does not apply in the $W^{+} W^{-}$case and nine, rather than six, independent helicity

\footnotetext{
${ }^{4}$ Angular distributions for two-particle decays of the $X$ bosons are given in the appendix.
} 
amplitudes are required to describe $X \rightarrow W^{+} W^{-}$decay. While the increase in the number of the helicity amplitudes implies that most of the formulas for angular distributions derived in this paper are not complete for $X \rightarrow W^{+} W^{-}$, it is interesting to remark that Eq. (2) remains the most general description of a spin-zero $X \rightarrow W^{+} W^{-}$decay and Eq. (35) is valid, with $R_{1}=R_{2}=1$.

Another aspect of the decay of the resonance $X$ to two non-identical particles is that forward-backward asymmetry can be generated. Of course, this requires that the initial state is asymmetric (e.g. $q \bar{q})$ as well. In general, the forward-backward asymmetry manifests itself in the odd terms in $\cos \theta^{*}$ distribution. To isolate and measure these terms, an unambiguous definition of the direction of the $z$-axis is required; a suggestion on how to do that was given in Ref. [43]. On the experimental side, the angular analysis of $X \rightarrow W^{+} W^{-} \rightarrow 4 l$ decay is challenging due to the presence of two neutrinos in the final state. It might be beneficial to consider semileptonic final states where one $W$ decays hadronically and the other leptonically. However, this case requires detailed studies because of potentially large irreducible backgrounds from events with jets and missing energy. Similar issues should be investigated if semileptonic final states are allowed in $X \rightarrow Z Z$ decays. We hope to return to the discussion of these channels in the near future.

Acknowledgments: We wish to thank Kaustubh Agashe for discussion of the KK graviton models. Several of us would like to thank CMS collaboration colleagues for feedback during the regular working group presentations of this analysis, and in particular Bob Cousins for discussion of the $\mathcal{S}$ significance estimator. This research is partially supported by US NSF under grants PHY-0644849, PHY-0758083, and PHY-0855365, by the A. P. Sloan Foundation, and by the University Research Association. FNAL is operated by Fermi Research Alliance, LLC under Contract No. DE-AC02-07CH11359 with the US DOE. We also acknowledge support by the start-up funds provided by the Johns Hopkins University (JHU). Calculations reported in this paper were performed on the Homewood High Performance Cluster of the JHU.

\section{Appendix A: Angular distributions for other decay channels}

In this appendix, the angular distributions for a resonance decaying into two particles, including $\gamma \gamma, g g, l^{+} l^{-}$, and $t \bar{t}$, are given. The general formula for the two-particle production and decay $g g$ or $q \bar{q} \rightarrow X \rightarrow P_{1} P_{2}$ can be obtained from Eq. (25) by changing the spin-quantization axis of the $X$ to $z$ and setting $\Omega=\left(\Phi_{1}, \theta^{*},-\Phi_{1}\right)$ and $\Omega^{*}=(0,0,0)$, where $\Phi_{1}$ is now an arbitrary azimuthal angle to be integrated out. The resulting formula reads

$$
\frac{d \Gamma\left(X_{J} \rightarrow P_{1} P_{2}\right)}{\Gamma d \cos \theta^{*}}=\left(J+\frac{1}{2}\right) \sum_{\lambda_{1}, \lambda_{2}} f_{\lambda_{1} \lambda_{2}} \sum_{m} f_{m}\left(d_{m, \lambda_{1}-\lambda_{2}}^{J}\left(\theta^{*}\right)\right)^{2}
$$

where $\lambda_{1}$ and $\lambda_{2}$ run over all possible helicities of $P_{1}$ and $P_{2}, m$ runs over all possible $X$ spin projections, and $f_{\lambda_{1} \lambda_{2}}=\left|A_{\lambda_{1} \lambda_{2}}\right|^{2} / \sum\left|A_{k l}\right|^{2}$, as defined earlier for the $X \rightarrow Z Z$ decay. However, this definition is more general and includes $\lambda_{i}= \pm 1 / 2$ for decays to fermions $X \rightarrow f \bar{f}$. In the latter case, we choose the same notation as in Eq. (29) for their parameterization with $f_{++}, f_{--}, f_{+-}, f_{-+}$, where we omit the $1 / 2$ for simplicity. Equation (A1) includes $f_{m}$ parameters, where $f_{0}=f_{z 0}, f_{ \pm 2}=f_{z 2} / 2$, and $f_{ \pm 1}=\left(f_{z 1} \pm \Delta f_{z 1}\right) / 2$, allowing for a quark direction measurement in $q \bar{q}$ production and, generally, for two non-identical particles $P_{1}$ and $P_{2}$.

We now specialize to particular channels. For the $X \rightarrow \gamma \gamma$ decay channel, the angular distribution can be obtained either from Eq. (A1) or by integrating out the $\Phi_{1}$ dependence in Eq. (33). Note that only $f_{++}, f_{--}$, and $f_{-+}=f_{+-}$ parameters are non-zero in the decay to two massless photons. Therefore

$$
\begin{aligned}
\frac{16 d \Gamma\left(X_{J=2} \rightarrow \gamma \gamma\right)}{5 \Gamma d \cos \theta^{*}}= & \left(2-2 f_{z 1}+f_{z 2}\right)-6\left(2-4 f_{z 1}-f_{z 2}\right) \cos ^{2} \theta^{*}+3\left(6-10 f_{z 1}-5 f_{z 2}\right) \cos ^{4} \theta^{*} \\
& +f_{+-}\left\{\left(2+2 f_{z 1}-7 f_{z 2}\right)+6\left(2-6 f_{z 1}+f_{z 2}\right) \cos ^{2} \theta^{*}-5\left(6-10 f_{z 1}-5 f_{z 2}\right) \cos ^{4} \theta^{*}\right\}
\end{aligned}
$$

This equation describes the most general case. The special case of the minimal coupling in both production and decay corresponds to $f_{z 1}+f_{z 2}=1$ and $f_{+-}=f_{-+}=1 / 2$. In this case, one obtains $\left(1+6 \cos ^{2} \theta^{*}+\cos ^{4} \theta^{*}\right)$ for the $g g$ production mechanism with $f_{z 2}=1$ and $\left(1-\cos ^{4} \theta^{*}\right)$ for the $q \bar{q}$ production mechanism with $f_{z 1}=1$. Equation (A2) is also applicable to the decay to two gluon jets $X_{J=2} \rightarrow g g$, but additional constraints could be used when the production and decay mechanisms are the same: $\left(1-f_{z 1}-f_{z 2}\right) / f_{z 2}=\left(1-f_{+-}-f_{-+}\right) /\left(f_{+-}+f_{-+}\right)$.

For the decay to a fermion-antifermion pair, we obtain

$$
\frac{8 d \Gamma\left(X_{J=1} \rightarrow f \bar{f}\right)}{3 \Gamma d \cos \theta^{*}}=\left(f_{+-}+f_{-+}\right)\left(1+\cos ^{2} \theta^{*}\right)+2\left(1-f_{+-}-f_{-+}\right)\left(1-\cos ^{2} \theta^{*}\right)+2\left(f_{+-}-f_{-+}\right) \Delta f_{z 1} \cos \theta^{*},
$$




$$
\begin{aligned}
\frac{16 d \Gamma\left(X_{J=2} \rightarrow f \bar{f}\right)}{5 \Gamma d \cos \theta^{*}}= & 2\left(f_{+-}+f_{-+}\right)\left\{\left(f_{z 1}+f_{z 2}\right)+3\left(2-3 f_{z 1}-2 f_{z 2}\right) \cos ^{2} \theta^{*}-\left(6-10 f_{z 1}-5 f_{z 2}\right) \cos ^{4} \theta^{*}\right\} \\
& +\left(1-f_{+-}-f_{-+}\right)\left\{\left(2-2 f_{z 1}+f_{z 2}\right)-6\left(2-4 f_{z 1}-f_{z 2}\right) \cos ^{2} \theta^{*}+3\left(6-10 f_{z 1}-5 f_{z 2}\right) \cos ^{4} \theta^{*}\right\} \\
& -4\left(f_{+-}-f_{-+}\right) \Delta f_{z 1}\left(\cos \theta^{*}-2 \cos ^{3} \theta^{*}\right) .
\end{aligned}
$$

where for a massless fermion in the final state $\left(f_{++}+f_{--}\right)=\left(1-f_{+-}-f_{-+}\right)=0$, which would describe the decay $X \rightarrow l^{+} l^{-}$. It follows from this formula that there is a forward-backward asymmetry in this decay, as was pointed out in Ref. [13] in the context of spin-one decays to a fermion pair. A dilution factor needs to be introduced in front of the $\Delta f_{z 1}$ terms, which depends on the ability to measure the sign of $\cos \theta^{*}$ in an experiment. The special case of the minimal coupling in gluon fusion corresponds to $f_{z 1}+f_{z 2}=1$.

\section{Appendix B: Supporting material}

Supporting material for this analysis may be found in Ref. [44], where we provide the Monte Carlo simulation program and the most general angular distributions used in this analysis. For completeness, we present the general angular distribution in the production and decay of a spin- $J$ particle $X$ in parton collisions $a b \rightarrow X \rightarrow Z Z \rightarrow$ $\left(f_{1} \bar{f}_{1}\right)\left(f_{2} \bar{f}_{2}\right)$. In order to simplify expressions, we redefine the fifth angle from $\Phi_{1}$ to $\Psi=\Phi_{1}+\Phi / 2$, which can be interpreted as the angle between the production plane and the average between the two decay planes shown in Fig. 11.

$$
\begin{aligned}
& \frac{\mathcal{N}_{J} d \Gamma_{J}}{\Gamma d \cos \theta^{*} d \Psi d \cos \theta_{1} d \cos \theta_{2} d \Phi}= \\
& F_{00}^{J}\left(\theta^{*}\right) \times\left\{4 f_{00} \sin ^{2} \theta_{1} \sin ^{2} \theta_{2}+\left(f_{++}+f_{--}\right)\left(\left(1+\cos ^{2} \theta_{1}\right)\left(1+\cos ^{2} \theta_{2}\right)+4 R_{1} R_{2} \cos \theta_{1} \cos \theta_{2}\right)\right. \\
& -2\left(f_{++}-f_{--}\right)\left(R_{1} \cos \theta_{1}\left(1+\cos ^{2} \theta_{2}\right)+R_{2}\left(1+\cos ^{2} \theta_{1}\right) \cos \theta_{2}\right) \\
& +4 \sqrt{f_{++} f_{00}}\left(R_{1}-\cos \theta_{1}\right) \sin \theta_{1}\left(R_{2}-\cos \theta_{2}\right) \sin \theta_{2} \cos \left(\Phi+\phi_{++}\right) \\
& +4 \sqrt{f_{--} f_{00}}\left(R_{1}+\cos \theta_{1}\right) \sin \theta_{1}\left(R_{2}+\cos \theta_{2}\right) \sin \theta_{2} \cos \left(\Phi-\phi_{--}\right) \\
& \left.+2 \sqrt{f_{++} f_{--}} \sin ^{2} \theta_{1} \sin ^{2} \theta_{2} \cos \left(2 \Phi+\phi_{++}-\phi_{--}\right)\right\} \\
& +4 F_{11}^{J}\left(\theta^{*}\right) \times\left\{\left(f_{+0}+f_{0-}\right)\left(1-\cos ^{2} \theta_{1} \cos ^{2} \theta_{2}\right)-\left(f_{+0}-f_{0-}\right)\left(R_{1} \cos \theta_{1} \sin ^{2} \theta_{2}+R_{2} \sin ^{2} \theta_{1} \cos \theta_{2}\right)\right. \\
& \left.+2 \sqrt{f_{+0} f_{0-}} \sin \theta_{1} \sin \theta_{2}\left(R_{1} R_{2}-\cos \theta_{1} \cos \theta_{2}\right) \cos \left(\Phi+\phi_{+0}-\phi_{0-}\right)\right\} \\
& +(-1)^{J} \times 4 F_{-11}^{J}\left(\theta^{*}\right) \times\left\{\left(f_{+0}+f_{0-}\right)\left(R_{1} R_{2}+\cos \theta_{1} \cos \theta_{2}\right)-\left(f_{+0}-f_{0-}\right)\left(R_{1} \cos \theta_{2}+R_{2} \cos \theta_{1}\right)\right. \\
& \left.+2 \sqrt{f_{+0} f_{0-}} \sin \theta_{1} \sin \theta_{2} \cos \left(\Phi+\phi_{+0}-\phi_{0-}\right)\right\} \sin \theta_{1} \sin \theta_{2} \cos (2 \Psi) \\
& +2 F_{22}^{J}\left(\theta^{*}\right) \times f_{+-}\left\{\left(1+\cos ^{2} \theta_{1}\right)\left(1+\cos ^{2} \theta_{2}\right)-4 R_{1} R_{2} \cos \theta_{1} \cos \theta_{2}\right\} \\
& +(-1)^{J} \times 2 F_{-22}^{J}\left(\theta^{*}\right) \times f_{+-} \sin ^{2} \theta_{1} \sin ^{2} \theta_{2} \cos (4 \Psi) \\
& +2 F_{02}^{J}\left(\theta^{*}\right) \times\left\{2 \sqrt{f_{00} f_{+-}} \sin \theta_{1} \sin \theta_{2} \times\left[\left(R_{1}-\cos \theta_{1}\right)\left(R_{2}+\cos \theta_{2}\right) \cos \left(2 \Psi-\phi_{+-}\right)\right.\right. \\
& \left.+\left(R_{1}+\cos \theta_{1}\right)\left(R_{2}-\cos \theta_{2}\right) \cos \left(2 \Psi+\phi_{+-}\right)\right] \\
& +\sqrt{f_{++} f_{+-}}\left[\sin ^{2} \theta_{1}\left(1-2 R_{2} \cos \theta_{2}+\cos ^{2} \theta_{2}\right) \cos \left(2 \Psi-\Phi+\phi_{+-}-\phi_{++}\right)\right. \\
& \left.+\left(1-2 R_{1} \cos \theta_{1}+\cos ^{2} \theta_{1}\right) \sin ^{2} \theta_{2} \cos \left(2 \Psi+\Phi-\phi_{+-}+\phi_{++}\right)\right] \\
& +\sqrt{f_{--} f_{+-}}\left[\sin ^{2} \theta_{1}\left(1+2 R_{2} \cos \theta_{2}+\cos ^{2} \theta_{2}\right) \cos \left(2 \Psi-\Phi-\phi_{+-}+\phi_{--}\right)\right. \\
& \left.\left.+\left(1+2 R_{1} \cos \theta_{1}+\cos ^{2} \theta_{1}\right) \sin ^{2} \theta_{2} \cos \left(2 \Psi+\Phi+\phi_{+-}-\phi_{--}\right)\right]\right\} \\
& -2 \sqrt{2} F_{01}^{J}\left(\theta^{*}\right) \times\left\{2 \sqrt { f _ { 0 0 } f _ { + 0 } } \left[\sin \theta_{1}\left(R_{1}-\cos \theta_{1}\right) \sin ^{2} \theta_{2} \cos \left(\Psi-\Phi / 2-\phi_{+0}\right)\right.\right. \\
& \left.-\sin ^{2} \theta_{1} \sin \theta_{2}\left(R_{2}-\cos \theta_{2}\right) \cos \left(\Psi+\Phi / 2+\phi_{+0}\right)\right] \\
& +2 \sqrt{f_{00} f_{0-}}\left[\sin ^{2} \theta_{1} \sin \theta_{2}\left(R_{2}+\cos \theta_{2}\right) \cos \left(\Psi+\Phi / 2-\phi_{0-}\right)\right.
\end{aligned}
$$




$$
\begin{aligned}
& \left.-\sin \theta_{1}\left(R_{1}+\cos \theta_{1}\right) \sin ^{2} \theta_{2} \cos \left(\Psi-\Phi / 2+\phi_{0-}\right)\right] \\
& +\sqrt{f_{++} f_{+0}}\left[\left(1-2 R_{1} \cos \theta_{1}+\cos ^{2} \theta_{1}\right) \sin \theta_{2}\left(R_{2}-\cos \theta_{2}\right) \cos \left(\Psi+\Phi / 2+\phi_{++}-\phi_{+0}\right)\right. \\
& \left.-\sin \theta_{1}\left(R_{1}-\cos \theta_{1}\right)\left(1-2 R_{2} \cos \theta_{2}+\cos ^{2} \theta_{2}\right) \cos \left(\Psi-\Phi / 2-\phi_{++}+\phi_{+0}\right)\right] \\
& +\sqrt{f_{++} f_{0-}}\left[\sin \theta_{1}\left(R_{1}-\cos \theta_{1}\right) \sin ^{2} \theta_{2} \cos \left(\Psi+3 \Phi / 2+\phi_{++}-\phi_{0-}\right)\right. \\
& \left.-\sin ^{2} \theta_{1} \sin \theta_{2}\left(R_{2}-\cos \theta_{2}\right) \cos \left(\Psi-3 \Phi / 2-\phi_{++}+\phi_{0-}\right)\right] \\
& +\sqrt{f_{--} f_{+0}}\left[\sin ^{2} \theta_{1} \sin \theta_{2}\left(R_{2}+\cos \theta_{2}\right) \cos \left(\Psi-3 \Phi / 2+\phi_{--}-\phi_{+0}\right)\right. \\
& \left.-\sin \theta_{1}\left(R_{1}+\cos \theta_{1}\right) \sin ^{2} \theta_{2} \cos \left(\Psi+3 \Phi / 2-\phi_{--}+\phi_{+0}\right)\right] \\
& +\sqrt{f_{--} f_{0-}}\left[\sin \theta_{1}\left(R_{1}+\cos \theta_{1}\right)\left(1+2 R_{2} \cos \theta_{2}+\cos ^{2} \theta_{2}\right) \cos \left(\Psi-\Phi / 2+\phi_{--}-\phi_{0-}\right)\right. \\
& \left.\left.-\left(1+2 R_{1} \cos \theta_{1}+\cos ^{2} \theta_{1}\right) \sin \theta_{2}\left(R_{2}+\cos \theta_{2}\right) \cos \left(\Psi+\Phi / 2-\phi_{--}+\phi_{0-}\right)\right]\right\} \\
& -2 \sqrt{2} F_{12}^{J}\left(\theta^{*}\right) \times\left\{\sqrt { f _ { + - } f _ { + 0 } } \left[\left(1-2 R_{1} \cos \theta_{1}+\cos ^{2} \theta_{1}\right) \sin \theta_{2}\left(R_{2}+\cos \theta_{2}\right) \cos \left(\Psi+\Phi / 2-\phi_{+-}+\phi_{+0}\right)\right.\right. \\
& \left.-\sin \theta_{1}\left(R_{1}+\cos \theta_{1}\right)\left(1-2 R_{2} \cos \theta_{2}+\cos ^{2} \theta_{2}\right) \cos \left(\Psi-\Phi / 2+\phi_{+-}-\phi_{+0}\right)\right] \\
& +\sqrt{f_{+-} f_{0-}}\left[\sin \theta_{1}\left(R_{1}-\cos \theta_{1}\right)\left(1+2 R_{2} \cos \theta_{2}+\cos ^{2} \theta_{2}\right) \cos \left(\Psi-\Phi / 2-\phi_{+-}+\phi_{0-}\right)\right. \\
& \left.\left.-\left(1+2 R_{1} \cos \theta_{1}+\cos ^{2} \theta_{1}\right) \sin \theta_{2}\left(R_{2}-\cos \theta_{2}\right) \cos \left(\Psi+\Phi / 2+\phi_{+-}-\phi_{0-}\right)\right]\right\} \\
& -(-1)^{J} \times 2 \sqrt{2} F_{-12}^{J}\left(\theta^{*}\right) \times\left\{\sqrt { f _ { + - } f _ { + 0 } } \left[\sin \theta_{1}\left(R_{1}-\cos \theta_{1}\right) \sin ^{2} \theta_{2} \cos \left(3 \Psi+\Phi / 2-\phi_{+-}+\phi_{+0}\right)\right.\right. \\
& \left.-\sin ^{2} \theta_{1} \sin \theta_{2}\left(R_{2}-\cos \theta_{2}\right) \cos \left(3 \Psi-\Phi / 2+\phi_{+-}-\phi_{+0}\right)\right] \\
& +\sqrt{f_{+-} f_{0-}}\left[\sin ^{2} \theta_{1} \sin \theta_{2}\left(R_{2}+\cos \theta_{2}\right) \cos \left(3 \Psi-\Phi / 2-\phi_{+-}+\phi_{0-}\right)\right. \\
& \left.\left.-\sin \theta_{1}\left(R_{1}+\cos \theta_{1}\right) \sin ^{2} \theta_{2} \cos \left(3 \Psi+\Phi / 2+\phi_{+-}-\phi_{0-}\right)\right]\right\}
\end{aligned}
$$

where $\mathcal{N}_{J}$ is the normalization factor which does not affect the angular distributions and the functions $F_{i j}^{J}\left(\theta^{*}\right)$ are defined as follows

$$
F_{i j}^{J}\left(\theta^{*}\right)=\sum_{m=0, \pm 1, \pm 2} f_{m} d_{m i}^{J}\left(\theta^{*}\right) d_{m j}^{J}\left(\theta^{*}\right),
$$

where $f_{ \pm 1}=f_{z 1} / 2, f_{ \pm 2}=f_{z 2} / 2$, and $f_{0}=f_{z 0}=\left(1-f_{z 1}-f_{z 2}\right)$. Note that for odd $J$ one has $f_{00}=f_{++}=f_{--}=0$, and therefore $F_{0 j}^{J=\text { odd }}\left(\theta^{*}\right)$ terms do not contribute.

Below we show $F_{i j}^{J}\left(\theta^{*}\right)$ explicitly for $J=0,1$, and 2 . For spin-zero, we have

$$
\begin{aligned}
& F_{00}^{0}=1, \\
& F_{11}^{0}=F_{-11}^{0}=F_{22}^{0}=F_{-22}^{0}=F_{02}^{0}=F_{01}^{0}=F_{12}^{0}=F_{-12}^{0}=0,
\end{aligned}
$$

where only four parameters $f_{++}, f_{--}, \phi_{++}$, and $\phi_{--}$remain relevant, and $f_{00}$ can be expressed as $f_{00}=\left(1-f_{++}-\right.$ $\left.f_{--}\right)$. For spin-one, we have

$$
\begin{aligned}
& F_{11}^{1}=\frac{1}{4}\left(1+\cos ^{2} \theta^{*}\right), \\
& F_{-11}^{1}=\frac{1}{4}\left(1-\cos ^{2} \theta^{*}\right), \\
& F_{00}^{1}=F_{22}^{1}=F_{-22}^{1}=F_{02}^{1}=F_{01}^{1}=F_{12}^{1}=F_{-12}^{1}=0,
\end{aligned}
$$

where only two parameters $f_{+0}$ and $\left[\phi_{+0}-\phi_{0-}\right]$ remain relevant, and $f_{0-}$ can be expressed as $f_{0-}=\left(1-2 f_{+0}\right) / 2$. Finally, for spin-two, we have

$$
F_{00}^{2}=\frac{1}{8}\left\{\left(2-2 f_{z 1}+f_{z 2}\right)-6\left(2-4 f_{z 1}-f_{z 2}\right) \cos ^{2} \theta^{*}+3\left(6-10 f_{z 1}-5 f_{z 2}\right) \cos ^{4} \theta^{*}\right\},
$$




$$
\begin{aligned}
& F_{11}^{2}=\frac{1}{4}\left\{\left(f_{z 1}+f_{z 2}\right)+3\left(2-3 f_{z 1}-2 f_{z 2}\right) \cos ^{2} \theta^{*}-\left(6-10 f_{z 1}-5 f_{z 2}\right) \cos ^{4} \theta^{*}\right\}, \\
& F_{-11}^{2}=-\frac{1}{4}\left\{\left(f_{z 1}-f_{z 2}\right)+\left(6-10 f_{z 1}-5 f_{z 2}\right) \cos ^{2} \theta^{*}\right\} \sin ^{2} \theta^{*}, \\
& F_{22}^{2}=\frac{1}{16}\left\{\left(6-2 f_{z 1}-5 f_{z 2}\right)-6\left(2-2 f_{z 1}-3 f_{z 2}\right) \cos ^{2} \theta^{*}+\left(6-10 f_{z 1}-5 f_{z 2}\right) \cos ^{4} \theta^{*}\right\}, \\
& F_{-22}^{2}=\frac{1}{16}\left\{6-10 f_{z 1}-5 f_{z 2}\right\} \sin ^{4} \theta^{*}, \\
& F_{02}^{2}=-\frac{1}{8} \sqrt{\frac{3}{2}}\left\{\left(2-2 f_{z 1}-3 f_{z 2}\right)-\left(6-10 f_{z 1}-5 f_{z 2}\right) \cos ^{2} \theta^{*}\right\} \sin ^{2} \theta^{*}, \\
& F_{01}^{2}=-\frac{\sqrt{6}}{8}\left\{\left(2-4 f_{z 1}-f_{z 2}\right)-\left(6-10 f_{z 1}-5 f_{z 2}\right) \cos ^{2} \theta^{*}\right\} \cos \theta^{*} \sin \theta^{*}, \\
& F_{12}^{2}=\frac{1}{8}\left\{\left(6-6 f_{z 1}-9 f_{z 2}\right)-\left(6-10 f_{z 1}-5 f_{z 2}\right) \cos ^{2} \theta^{*}\right\} \cos \theta^{*} \sin \theta^{*}, \\
& F_{-12}^{2}=-\frac{1}{8}\left(6-10 f_{z 1}-5 f_{z 2}\right) \cos \theta^{*} \sin ^{3} \theta^{*},
\end{aligned}
$$

where all parameters contribute and again $f_{00}$ can be expressed as $f_{00}=\left(1-f_{++}-f_{--}-2 f_{+0}-2 f_{0-}-2 f_{+-}\right)$.

[1] L. Evans and P. Bryant (editors), JINST 3 S08001 (2008).

[2] ATLAS Collaboration, JINST 3 S08003 (2008).

[3] CMS Collaboration, JINST 3 S08004 (2008).

[4] Tevatron Electroweak Working Group, CDF and D0 Collaborations, preprint arXiv:0808.1089 [hep-ex].

[5] For review of $B$-physics polarization measurements see A. V. Gritsan and J. G. Smith, page 910 of Particle Data Group, Phys. Lett. B 667, 1 (2008).

[6] A. Soni and R. M. Xu, Phys. Rev. D 48, 5259 (1993).

[7] S.Y. Choi, D. J. Miller, M.M. Muhlleitner, and P. M. Zerwas, Phys. Lett. B553, 61 (2003).

[8] C.P. Buszello, I. Fleck, P. Marquard, and J.J. van der Bij, Eur. Phys. J. C32, 209 (2004).

[9] W. Y. Keung, I. Low, and J. Shu, Phys. Rev. Lett. 101, 091802 (2008).

[10] O. Antipin and A. Soni, JHEP 0810, 018 (2008).

[11] K. Hagiwara, Q. Li, and K. Mawatari, preprint arXiv:0905.4314 [hep-ph] (2009).

[12] Q.-H. Cao, et al., preprint arXiv:0911.3398 [hep-ph].

[13] J. L. Rosner, Phys. Rev. D 54, 1078 (1996).

[14] H. Davoudiasl, J. L. Hewett, and T. G. Rizzo, Phys. Rev. Lett. 84, 2080 (2000); Phys. Rev. D 63, 075004 (2001).

[15] B. C. Allanach, K. Odagiri, M. A. Parker, and B. R. Webber, JHEP 0009, 019 (2000).

[16] B. C. Allanach et al., JHEP 0212, 039 (2002).

[17] R. Cousins, J. Mumford, J. Tucker, and V. Valuev, JHEP 11, 046 (2005).

[18] P. Osland, A. A. Pankov, N. Paver, and A. V. Tsytrinov, Phys. Rev. D 78, 035008 (2008); arXiv:0902.1593 [hep-ph].

[19] For review of Higgs studies see G. Bernardi, M. Carena, and T. Junk, page 414 of Particle Data Group, Phys. Lett. B 667, 1 (2008).

[20] L. Randall and R. Sundrum, Phys. Rev. Lett. 83, 3370 (1999).

[21] A. L. Fitzpatrick, J. Kaplan, L. Randall, and L.-T. Wang JHEP 0709, 013 (2007).

[22] K. Agashe, H. Davoudiasl, G. Perez, and A. Soni, Phys. Rev. D 76, 036006 (2007).

[23] O. Antipin, D. Atwood, and A. Soni, Phys. Lett. B666, 155 (2008).

[24] J. E. Juknevich, D. Melnikov, and M. J. Strassler, JHEP 0907, 055 (2009).

[25] V. Barger, Y. Gao, and W.-Y. Keung, Phys. Lett. B655, 228 (2007).

[26] T. L. Trueman, Phys. Rev. D 18, 3423 (1978).

[27] J. R. Dell'Aquila and C. A. Nelson, Phys. Rev. D 33, 80 (1986).

[28] M. R. Buckley, S. Y. Choi, K. Mawatari, and H. Murayama, Phys. Lett. B 672, 275 (2009).

[29] H. Murayama and V. Rentala, preprint arXiv:0904.4561 [hep-ph].

[30] G. Kramer and W.F. Palmer, Phys. Rev. D 45, 193 (1992).

[31] A. Datta et al., Phys. Rev. D 77, 114025 (2008).

[32] J. C. Collins and D. E. Soper, Phys. Rev. D 16, 2219 (1977)

[33] K. Hagiwara, J. Kanzaki, Q. Li, and K. Mawatari, Eur. Phys. J. C 56, 435 (2008).

[34] ATLAS Collaboration, preprint arXiv:0901.0512 [hep-ex] (2009).

[35] CMS Collaboration, preprint CMS PAS HIG-08-003 (2009).

[36] J. Alwall et al., JHEP 0709, 028 (2007).

[37] J. Pumplin et al.,, JHEP 0207, 012 (2002). 
[38] P. M. Nadolsky et al., Phys. Rev. D 78, 013004 (2008).

[39] T. Sjostrand, S. Mrenna, and P. Skands, JHEP 05, 026 (2006).

[40] CMS Collaboration, preprint arXiv:0910.2505 [physics.ins-det], to appear in JINST (2010).

[41] BABAR Collaboration, B. Aubert et al., Phys. Rev. D 78, 092008 (2008).

[42] F. James et al., MINUIT, "Function minimization and error analysis CERN program library;" R. Brun et al., "Root data-analysis framework;" and D. Kirkby et al., "The RooFit toolkit for data modeling," http://root.cern.ch/

[43] M. Dittmar, Phys. Rev. D 55, 161 (1997).

[44] See documentation at http://www.pha.jhu.edu/spin/ 